\title{
Evaluation and interpolation over multivariate skew polynomial rings
}

\author{
Umberto Martínez-Peñas ${ }^{* 1,2}$ and Frank R. Kschischang ${ }^{\dagger 1}$ \\ ${ }^{1}$ Dept. of Electrical \& Computer Engineering, University of Toronto, Canada \\ ${ }^{2}$ Dept. of Mathematical Sciences, Aalborg University, Denmark
}

\begin{abstract}
The concepts of evaluation and interpolation are extended from univariate skew polynomials to multivariate skew polynomials, with coefficients over division rings. Iterated skew polynomial rings are in general not suitable for this purpose. Instead, multivariate skew polynomial rings are constructed in this work as follows: First, free multivariate skew polynomial rings are defined, where multiplication is additive on degrees and restricts to concatenation for monomials. This allows to define the evaluation of any skew polynomial at any point by unique remainder division. Multivariate skew polynomial rings are then defined as the quotient of the free ring by (two-sided) ideals that vanish at every point. The main objectives and results of this work are descriptions of the sets of zeros of these multivariate skew polynomials, the families of functions that such skew polynomials define, and how to perform Lagrange interpolation with them. To obtain these descriptions, the existing concepts of P-closed sets, P-independence, P-bases (which are shown to form a matroid) and skew Vandermonde matrices are extended from the univariate case to the multivariate one.
\end{abstract}

Keywords: Derivations, free polynomial rings, Lagrange interpolation, Newton interpolation, skew polynomials, Vandermonde matrices.

MSC: 08B20, 11C08, 12E10.

\section{Introduction}

Univariate skew polynomial rings, introduced in [20, are those "non-commutative polynomial rings", over some coefficient ring, whose addition is the usual one, but whose multiplication is arbitrary with the following restrictions: The $i$ th power (being $i$ a natural number) of the variable $x$ corresponds to the monomial " $x^{i}$ ", and the degree of a product of two arbitrary polynomials is the sum of their degrees. Adding the commutativity property yields the conventional polynomial ring, that is, the monoid ring of the natural numbers over the coefficient ring.

*umberto@ece.utoronto.ca

${ }^{\dagger}$ frank@ece.utoronto.ca 
An extension of the concept of evaluation to skew polynomials over division rings was first given in [10] and further developed in [11, 12]. Since a skew polynomial ring (over a division ring) is a right-Euclidean domain [20], the evaluation of $F(x)$ on a point $a$ is defined in [10, 12] as the remainder of the Euclidean division of $F(x)$ by $x-a$ on the right. Such extension is natural in the sense that it is based on the "Remainder Theorem" for conventional polynomials and it is analogous to projecting on a quotient ring defined by a maximal ideal, as in algebraic geometry. This concept of evaluation helps unify the study of Vandermonde, Moore and Wronskian matrices [10, 12] and further matrix types (see [19, p. 604] for instance), and gives a natural framework for Hilbert 90 Theorems [13] and pseudolinear transformations [15], which unify semilinear and differential transformations (see also [3, Sec. 8.4]). It has also provided errorcorrecting codes with good minimum Hamming distance [2, maximum rank distance codes [5], and maximum sum-rank distance codes [19] with finite-field sizes that are not exponential in the code length, in constrast with [5] (see [19, Sec. 4.2]).

Extending this concept of evaluation to multivariate skew polynomials is not straightforward. In general, unique remainder algorithms [14, Sec. 4] do not hold for iterated skew polynomials since they do not satisfy Jategaonkar's condition [8] for $n>1$ variables (see [14, Prop. 4.7] and also [3, Sec. 8.8]). Recently in [7, it is proposed to evaluate certain iterated skew polynomials (those forming Poincaré-Birkhoff-Witt extensions following [23, Def 2.1]) at points $\left(a_{1}, a_{2}, \ldots, a_{n}\right)$ where $x_{1}-a_{1}, x_{2}-a_{2}, \ldots, x_{n}-a_{n}$ form a Göbner-Shirshov basis, since then a unique remainder algorithm exists. However, this does not include all iterated skew polynomial rings or affine points (see [7, Ex. 3.5]) and the important concepts and results from [10, 11, 12] do not seem to hold.

In this work, we overcome these issues by considering an alternative construction. We start by defining free multivariate skew polynomial rings (using the free monoid with basis $x_{1}, x_{2}, \ldots, x_{n}$ ) following Ore's idea: The product of two monomials consists in appending them, and the degree of a product of two skew polynomials is the sum of their degrees. Over fields, adding commutativity between constants and variables (that is, turning the ring into an algebra) yields the conventional free algebra [․ Sec. 0.11] as a particular case. Thanks to this definition, we show that we may define the evaluation of any (free) skew polynomial $F\left(x_{1}, x_{2}, \ldots, x_{n}\right)$ at any affine point $\left(a_{1}, a_{2}, \ldots, a_{n}\right)$ as the unique remainder of the Euclidean division of $F\left(x_{1}, x_{2}, \ldots, x_{n}\right)$ by $x_{1}-a_{1}, x_{2}-a_{2}$, $\ldots, x_{n}-a_{n}$ on the right. Once this is done, we may define general (nonfree) skew polynomial rings, where evaluation is still natural at every point, as quotients of the free ring by two-sided ideals of skew polynomials that vanish at every point (Definition 19). Reasonably behaved iterated skew polynomial rings are also quotients of the introduced free multivariate skew polynomial rings, and evaluations by unique remainder division as in [7] are recovered by the proposed evaluations (Remark 21), although the converse does not seem to hold.

Our main objective is to describe the functions obtained by evaluating multivariate skew polynomials over division rings, under some finiteness conditions (see the paragraph below). This problem is closely related to that of interpolation in the sense of Lagrange, which has been studied previously in the univariate case in [4, 10, 12, 16, 22]. 
Our main results are as follows: We obtain a description of the family of such functions, when defined on a finitely generated set of zeros (P-closed set), as a left vector space over the division ring of coefficients, and we find its dimension and a left basis (Theorem 5). For this, we first obtain a Lagrange-type interpolation theorem (Theorem 4) on P-closed sets. To this end, we need to extend first the concept of $P$-independence and P-basis from [11, Sec. 4], which naturally form a matroid (Proposition 27). See 21] for more details on matroid theory. For that purpose, we need to introduce ideals of zeros, whose properties are based on extensions to the multivariate case of tools from [10, 11, 12]: A multiplication that is additive on degrees (Theorem 11), an iterative evaluation on monomials (Theorem 2) and a product rule (Theorem 3).

Apart from its own interest, our main motivations to develop this theory come from the theory of error-correcting codes over finite fields, in view of [2, 5, 19], as explained above. A definition of skew Reed-Muller codes has been recently proposed [7, based on evaluating certain iterated skew polynomials at certain points as noted previously. However, the core properties of skew polynomial evaluation codes rely on the matroid given by P-independence and evaluation on P-bases (see [19]), which we introduce in the multivariate case in this work. Apart from applications in coding theory, it has been recently shown in [18] that Hilbert's Theorem 90 can be naturally stated and proven using the framework of this paper for general Galois extensions of fields (as considered by Noether) using arbitrary generators and relations of the Galois group (note that univariate skew polynomials restrict Hilbert 90 Theorems to a single generator [13], as originally stated by Kummer and Hilbert). A differential or more general version of such a Hilbert's Theorem 90 can be similarly put in this framework. Further applications in Galois theory or partial differential equations (such as a study of multivariate Moore or Wronskian matrices) may be possible and of interest.

The organization is as follows. In Section 2, we show which multiplications are additive on degrees over "free multivariate polynomial rings" (Theorem 1), extending [20. Eq. (3), (4) \& (5)]. In Section 3, we show how to define evaluations as remainders of Euclidean divisions and give a recursive formula for monomials (Theorem 2), extending [12, Lemma 2.4] and [12, Eq. (2.3)]. In Section 4, we show how the product of two skew polynomials is preserved after evaluation (Theorem [3), extending [12, Th. 2.7]. In Section 5, we define P-closed sets and ideals of zeros, and give their basic properties. Using them, we define in Section 6 nonfree multivariate skew polynomial rings (Definition 19). In Section 7, we extend the crucial concepts of P-independence and P-bases from [11, Sec. 4] to our context. In Section 8 , we show the existence of Lagrange interpolating skew polynomials (Theorem 4). In Section 9, we obtain the dimension and left bases of the left vector space of skew polynomial functions over a finitely generated P-closed set (Theorem [5). In Section 10, we give explicit computational methods to find such dimensions and bases and to perform Lagrange interpolation, via an extension of the Vandermonde matrices considered in [10, 12. The complexity for finding ranks and Pbases is exponential in general, but given a P-basis, the complexity of finding Lagrange interpolating polynomials is polynomial. 


\section{Notation}

Unless otherwise stated, $\mathbb{F}$ will denote a division ring. Assuming $\mathbb{F}$ to be finite (thus a field [17]) avoids all other finiteness assumptions.

For positive integers $m$ and $n, \mathbb{F}^{m \times n}$ will denote the set of $m \times n$ matrices over $\mathbb{F}$, and $\mathbb{F}^{n}$ will denote the set of column vectors of length $n$ over $\mathbb{F}$. That is, $\mathbb{F}^{n}=\mathbb{F}^{n \times 1}$.

On a non-commutative ring $\mathcal{R}$, we will denote by $(A) \subseteq \mathcal{R}$ the left ideal generated by a set $A \subseteq \mathcal{R}$, and on a left vector space $\mathcal{V}$ over $\mathbb{F}$, we will denote by $\langle B\rangle \subseteq \mathcal{V}$ the $\mathbb{F}$-linear left vector space generated by a set $B \subseteq \mathcal{V}$. We use the simplified notation $\left(F_{1}, F_{2}, \ldots, F_{n}\right)=\left(\left\{F_{1}, F_{2}, \ldots, F_{n}\right\}\right)$ and $\left\langle F_{1}, F_{2}, \ldots, F_{n}\right\rangle=\left\langle\left\{F_{1}, F_{2}, \ldots, F_{n}\right\}\right\rangle$.

All rings in this work will be assumed to have multiplicative identity.

\section{Free skew polynomial rings, matrix morphisms and vec- tor derivations}

In this section, we show which multiplications over a free non-commutative polynomial ring consist in appending monomials and are additive on degrees. See Remark 7 to see why we cannot assume that variables commute with each other, unless we are dealing with conventional multivariate polynomials over fields. See Remarks 8 and 21 to see why we do not consider iterated skew polynomial rings.

Fix a positive integer $n$ from now on, let $x_{1}, x_{2}, \ldots, x_{n}$ be $n$ distinct characters, and denote by $\mathcal{M}$ the set of all finite strings using these characters, that is, the free monoid with basis $x_{1}, x_{2}, \ldots, x_{n}$ (see [3, Sec. 6.5]). The empy string will be denoted by 1 . A character $x_{i}$ will be called a variable, an element $\mathfrak{m} \in \mathcal{M}$ will be called a monomial, and we will define its degree, denoted by $\operatorname{deg}(\mathfrak{m})$, as its length as a string.

Let $\mathcal{R}$ be the left vector space over $\mathbb{F}$ with basis $\mathcal{M}$. That is, every element $F \in \mathcal{R}$ can be expressed uniquely as a linear combination (with coefficients on the left)

$$
F=\sum_{\mathfrak{m} \in \mathcal{M}} F_{\mathfrak{m}} \mathfrak{m}
$$

where $F_{\mathfrak{m}} \in \mathbb{F}$, for $\mathfrak{m} \in \mathcal{M}$, and $F_{\mathfrak{m}}=0$ except for a finite number of monomials.

An element $F \in \mathcal{R}$ will be called a (multivariate) skew polynomial, and we will define its degree, denoted by $\operatorname{deg}(F)$, as the maximum degree of a monomial $\mathfrak{m} \in \mathcal{M}$ such that $F_{\mathfrak{m}} \neq 0$, if $F \neq 0$. We will define $\operatorname{deg}(F)=\infty$ if $F=0$.

Formally, our objective is to provide $\mathcal{R}$ with an inner product $\mathcal{R} \times \mathcal{R} \longrightarrow \mathcal{R}$ that turns it into a non-commutative ring with 1 as multiplicative identity, restricts to the operation $\mathcal{M} \times \mathcal{M} \longrightarrow \mathcal{M}$ that consists in appending strings, and where the degree of a product of two skew polynomials is the sum of their degrees.

First observe that, by identifying $a \in \mathbb{F}$ with $a 1 \in \mathcal{R}$, we may assume that $\mathbb{F} \subseteq \mathcal{R}$, with the elements in $\mathbb{F}$ called constants. Furthermore, $\mathbb{F}$ is a subring of $\mathcal{R}$ as long as 1 is the multiplicative identity. Next, by inspecting constants and variables, we see that we need functions

$$
\sigma_{i, j}: \mathbb{F} \longrightarrow \mathbb{F}, \quad \text { and } \quad \delta_{i}: \mathbb{F} \longrightarrow \mathbb{F}
$$


for $i, j=1,2, \ldots, n$, such that

$$
x_{i} a=\sum_{j=1}^{n} \sigma_{i, j}(a) x_{j}+\delta_{i}(a),
$$

for $i=1,2, \ldots, n$, and for all $a \in \mathbb{F}$. This defines two maps

$$
\sigma: \mathbb{F} \longrightarrow \mathbb{F}^{n \times n}: a \mapsto\left(\begin{array}{cccc}
\sigma_{1,1}(a) & \sigma_{1,2}(a) & \ldots & \sigma_{1, n}(a) \\
\sigma_{2,1}(a) & \sigma_{2,2}(a) & \ldots & \sigma_{2, n}(a) \\
\vdots & \vdots & \ddots & \vdots \\
\sigma_{n, 1}(a) & \sigma_{n, 2}(a) & \ldots & \sigma_{n, n}(a)
\end{array}\right)
$$

and

$$
\delta: \mathbb{F} \longrightarrow \mathbb{F}^{n}: a \mapsto\left(\begin{array}{c}
\delta_{1}(a) \\
\delta_{2}(a) \\
\vdots \\
\delta_{n}(a)
\end{array}\right)
$$

With this more compact notation, we may write Equation (11) as

$$
\mathbf{x} a=\sigma(a) \mathbf{x}+\delta(a),
$$

where $\mathbf{x}$ is a column vector containing $x_{i}$ in the $i$ th row, for $i=1,2, \ldots, n$. We have the following result, which extends the discussion in the case $n=1$ given at the beginning of [20]. See also [3, Th. 10.1].

Theorem 1. If an inner product in $\mathcal{R}$ turns it into a non-commutative ring with multiplicative identity 1 , consists in appending monomials when restricted to $\mathcal{M}$ and is additive on degrees, then it is given on constants and variables as in (1), the map

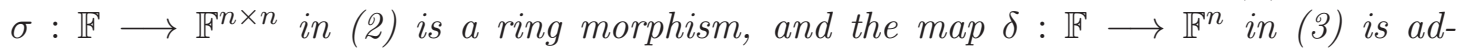
ditive and satisfies that

$$
\delta(a b)=\sigma(a) \delta(b)+\delta(a) b,
$$

for all $a, b \in \mathbb{F}$.

Conversely, for any two such maps $\sigma: \mathbb{F} \longrightarrow \mathbb{F}^{n \times n}$ and $\delta: \mathbb{F} \longrightarrow \mathbb{F}^{n}$, there exists a unique inner product in $\mathcal{R}$ satisfying the properties in the previous paragraph. Furthermore, two such inner products are equal if, and only if, the corresponding maps are equal.

Proof. First assume that a given inner product in $\mathcal{R}$ satisfies the properties given in the first paragraph. The additive properties of $\sigma$ and $\delta$ then follow from

$$
x_{i}(a+b)=\left(x_{i} a\right)+\left(x_{i} b\right),
$$

for all $a, b \in \mathbb{F}$ and all $i=1,2, \ldots, n$, their multiplicative properties follow from

$$
x_{i}(a b)=\left(x_{i} a\right) b,
$$


for all $a, b \in \mathbb{F}$ and all $i=1,2, \ldots, n$, and $\sigma(1)=I$ follows from $x_{i} 1=1 x_{i}$ (since 1 is a multiplicative identity) for all $i=1,2, \ldots, n$.

Next, the uniqueness and equality properties in the second paragraph are straightforward using Equations (10) or (4).

Finally, given a ring morphism $\sigma: \mathbb{F} \longrightarrow \mathbb{F}^{n \times n}$ and an additive map $\delta: \mathbb{F} \longrightarrow \mathbb{F}^{n}$ satisfying (5), we may define the desired inner product in $\mathcal{R}$ as follows.

First, constants in $\mathbb{F}$ act on the left as scalars $((a 1) F=a F$, for all $F \in \mathcal{R})$. Now given $\mathfrak{m}, \mathfrak{n} \in \mathcal{M}$, we define recursively on $\mathfrak{m}$ the products

$$
\left(\mathfrak{m} x_{i}\right)(a \mathfrak{n})=\sum_{j=1}^{n} \mathfrak{m}\left(\sigma_{i, j}(a)\left(x_{j} \mathfrak{n}\right)\right)+\mathfrak{m}\left(\delta_{i}(a) \mathfrak{n}\right),
$$

for all $i=1,2, \ldots, n$ and all $a \in \mathbb{F}$, where $\mathfrak{m} x_{i}$ and $x_{j} \mathfrak{n}$ denote appending of monomials. Observe that this already defines, recursively on $\mathfrak{m}$, the products of monomials as appending them, by choosing $a=1$.

Finally, given general skew polynomials $F=\sum_{\mathfrak{m} \in \mathcal{M}} F_{\mathfrak{m}} \mathfrak{m}$ and $G=\sum_{\mathfrak{n} \in \mathcal{M}} G_{\mathfrak{n}} \mathfrak{n}$, where $F_{\mathfrak{m}}, G_{\mathfrak{m}} \in \mathcal{R}$, for all $\mathfrak{m} \in \mathcal{M}$, we define

$$
F G=\sum_{\mathfrak{m} \in \mathcal{M}} \sum_{\mathfrak{n} \in \mathcal{M}} F_{\mathfrak{m}}\left(\mathfrak{m}\left(G_{\mathfrak{n}} \mathfrak{n}\right)\right) .
$$

Note that this product is well-defined, since $\operatorname{deg}(F)=d$ and $\operatorname{deg}(G)=e$ imply that the coefficient of $\mathfrak{m}$ in $F G$ is zero whenever $\operatorname{deg}(\mathfrak{m})>d+e$, for all $F, G \in \mathcal{R}$ and all $\mathfrak{m} \in \mathcal{M}$. The properties of such an inner product stated in the theorem are all trivial, except for associativity, whose verification is left to the reader.

This motivates the following definitions:

Definition 1 (Matrix morphisms and vector derivations). We call every ring morphism $\sigma: \mathbb{F} \longrightarrow \mathbb{F}^{n \times n}$ a matrix morphism (over $\mathbb{F}$ ), and we say that a map $\delta: \mathbb{F} \longrightarrow$ $\mathbb{F}^{n}$ is a $\sigma$-vector derivation (over $\mathbb{F}$ ) if it is additive and satisfies

$$
\delta(a b)=\sigma(a) \delta(b)+\delta(a) b,
$$

for all $a, b \in \mathbb{F}$.

Definition 2 (Free multivariate skew polynomial rings). Given a matrix morphism $\sigma: \mathbb{F} \longrightarrow \mathbb{F}^{n \times n}$ and a $\sigma$-vector derivation $\delta: \mathbb{F} \longrightarrow \mathbb{F}^{n}$, we define the free (multivariate) skew polynomial ring corresponding to $\sigma$ and $\delta$ as the unique ring $\mathcal{R}=\mathbb{F}[\mathbf{x} ; \sigma, \delta]$ with the inner product given by (11).

Observe that the conventional free multivariate polynomial ring (called free algebra over $\mathbb{F}$ when $\mathbb{F}$ is commutative, see [3, Sec. 0.11] and [3, Sec. 6.5]) on the variables $x_{1}, x_{2}, \ldots, x_{n}$ is obtained by choosing $\sigma=\operatorname{Id}$ and $\delta=0$, where we define $\operatorname{Id}(a)=a I$, for all $a \in \mathbb{F}$. Moreover, observe that this is the only case where constants and variables commute, which coincides with the only case where $\mathbb{F}[\mathbf{x} ; \sigma, \delta]$ is an algebra over $\mathbb{F}$ when 
$\mathbb{F}$ is commutative (here by algebra we mean a ring $\mathcal{R}$ that is a vector space over $\mathbb{F}$ and whose inner product is $\mathbb{F}$-bilinear, as in [3]). Finally, observe also that $\mathbb{F}[\mathbf{x} ; \sigma, \delta]$ can still be characterized by a universal property similar to that of the free algebra. We only need to replace in the universal property the commutativity of constants and variables on free algebras by the rule (11). We leave the details to the reader.

We conclude the section with some particular instances of matrix morphisms and vector derivations of interest:

Example 3. A matrix morphism $\sigma: \mathbb{F} \longrightarrow \mathbb{F}^{n \times n}$ satisfies $\sigma_{i, j}(a)=0$, for all $a \in \mathbb{F}$ and all $i \neq j$ if, and only if, there exist ring endomorphisms $\sigma_{i}: \mathbb{F} \longrightarrow \mathbb{F}$, for $i=1,2, \ldots, n$, such that

$$
\sigma(a)=\left(\begin{array}{cccc}
\sigma_{1}(a) & 0 & \ldots & 0 \\
0 & \sigma_{2}(a) & \ldots & 0 \\
\vdots & \vdots & \ddots & \vdots \\
0 & 0 & \ldots & \sigma_{n}(a)
\end{array}\right)
$$

for all $a \in \mathbb{F}$. It is trivial to check that the family of $\sigma$-vector derivations in this case are precisely those such that $\delta_{i}$ is a $\sigma_{i}$-derivation, for $i=1,2, \ldots, n$. An example is $\mathbb{F}=$ $k\left(t_{1}, t_{2}, \ldots, t_{n}\right)$, where $k$ is a field, $t_{1}, t_{2}, \ldots, t_{n}$ are algebraically independent variables, $\sigma_{i}=\mathrm{Id}$ and $\delta_{i}=\frac{\partial}{\partial t_{i}}$ is the conventional $i$ th partial derivative, for $i=1,2, \ldots, n$.

Example 4. Let $\sigma: \mathbb{F} \longrightarrow \mathbb{F}^{n \times n}$ be a matrix morphism, and let $\boldsymbol{\beta} \in \mathbb{F}^{n}$. The map $\delta: \mathbb{F} \longrightarrow \mathbb{F}^{n}$ defined by

$$
\delta(a)=\sigma(a) \boldsymbol{\beta}-\boldsymbol{\beta} a,
$$

for all $a \in \mathbb{F}$, is a $\sigma$-vector derivation. When $n=1$, these vector derivations are called inner derivations in the literature.

\section{Evaluations of multivariate skew polynomials}

In this section, we show how to define evaluation maps $E_{\mathbf{a}}: \mathbb{F}[\mathbf{x} ; \sigma, \delta] \longrightarrow \mathbb{F}$, for all $\mathbf{a} \in \mathbb{F}^{n}$, that can be considered natural or standard. We will first require that these maps are left linear forms over $\mathbb{F}$. We may then define the total evaluation map as

$$
E: \mathbb{F}[\mathbf{x} ; \sigma, \delta] \longrightarrow \mathbb{F}^{\mathbb{F}^{n}}: F \mapsto\left(E_{\mathbf{a}}(F)\right)_{\mathbf{a} \in \mathbb{F}^{n}},
$$

which is again left linear. By linearity, we have that

$$
E_{\mathbf{a}}\left(\sum_{\mathfrak{m} \in \mathcal{M}} F_{\mathfrak{m}} \mathfrak{m}\right)=\sum_{\mathfrak{m} \in \mathcal{M}} F_{\mathfrak{m}} N_{\mathfrak{m}}(\mathbf{a}),
$$

for all $\mathbf{a} \in \mathbb{F}^{n}$, all $F_{\mathfrak{m}} \in \mathbb{F}$, and for functions

$$
N_{\mathfrak{m}}: \mathbb{F}^{n} \longrightarrow \mathbb{F}: \mathbf{a} \longrightarrow E_{\mathbf{a}}(\mathfrak{m}),
$$

where $\mathfrak{m} \in \mathcal{M}$. Therefore, giving a total evaluation map $E$ is equivalent to giving the family of functions $\left(N_{\mathfrak{m}}\right)_{\mathfrak{m} \in \mathcal{M}}$, thus these will be called fundamental functions of the 
evaluation $E$. When $n=1$, the fundamental functions $N_{i}=N_{x^{i}}$, for $i=0,1,2, \ldots$, coincide with those in [10, 12.

As stated in Section 1, a standard way of understanding evaluations of multivariate conventional polynomials is by giving a canonical ring isomorphism

$$
\mathbb{F}\left[x_{1}, x_{2}, \ldots, x_{n}\right] /\left(x_{1}-a_{1}, x_{2}-a_{2}, \ldots, x_{n}-a_{n}\right) \longrightarrow \mathbb{F},
$$

for all $a_{1}, a_{2}, \ldots, a_{n} \in \mathbb{F}$, due to the "Remainder Theorem". The same idea is used in classical algebraic geometry to define evaluations as projections to a quotient ring given by a maximal ideal, which would be isomorphic to the so-called residue field.

To obtain such an isomorphism, we give a Euclidean-type division for skew polynomials of the type $x_{1}-a_{1}, x_{2}-a_{2}, \ldots, x_{n}-a_{n}$ :

Lemma 5. For any $a_{1}, a_{2}, \ldots, a_{n} \in \mathbb{F}$ and any $F \in \mathbb{F}[\mathbf{x} ; \sigma, \delta]$, there exist unique $G_{1}, G_{2}, \ldots, G_{n} \in \mathbb{F}[\mathbf{x} ; \sigma, \delta]$ and $b \in \mathbb{F}$ such that

$$
F=\sum_{i=1}^{n} G_{i}\left(x_{i}-a_{i}\right)+b .
$$

Proof. Existence is proven by a Euclidean division algorithm as usual. We next prove the uniqueness property. We only need to prove that if

$$
\sum_{i=1}^{n} G_{i}\left(x_{i}-a_{i}\right)+b=0,
$$

then $G_{1}=G_{2}=\ldots=G_{n}=b=0$. Assume the opposite. Without loss of generality, we may assume that $G_{n} \neq 0$ and $\operatorname{deg}\left(G_{n}\right) \geq \operatorname{deg}\left(G_{i}\right)$, for all $i$ with $G_{i} \neq 0$.

Let $\prec$ denote the graded lexicographic (from right to left) ordering in $\mathcal{M}$ with $x_{1} \prec$ $x_{2} \prec \ldots \prec x_{n}$, and denote by $\operatorname{LM}(G) \in \mathcal{M}$ the leading monomial of a skew polynomial $G \in \mathbb{F}[\mathbf{x} ; \sigma, \delta]$ with respect to $\prec$. Then we see that the monomial $\operatorname{LM}\left(G_{n}\left(x_{n}-a_{n}\right)\right)=$ $\operatorname{LM}\left(G_{n}\right) x_{n}$ cannot be cancelled by any other monomial on the left-hand side of (8). This is absurd and thus $G_{i}=0$, for all $i=1,2, \ldots, n$. Hence $b=0$ and we are done.

Remark 6. Observe that the facts that the product in $\mathbb{F}[\mathbf{x} ; \sigma, \delta]$ consists in appending monomials and is additive on degrees are crucial in the proof of the previous lemma, since they allow us to state that $\operatorname{LM}\left(G_{n}\left(x_{n}-a_{n}\right)\right)=\operatorname{LM}\left(G_{n}\right) x_{n}$ for the graded lexicographic ordering. These properties also ensure that the division algorithm does not run indefinitely. Note moreover that $\mathbb{F}$ can be an arbitrary ring, since the leading coefficients of $x_{1}-a_{1}, x_{2}-a_{2}, \ldots, x_{n}-a_{n}$ are all 1 .

Remark 7. Observe that (being $\mathbb{F}$ a division ring) we cannot guarantee that Lemma 5 (uniqueness of remainders) holds if we allow the variables to commute, unless we are dealing with multivariate conventional polynomials over fields.

Assume that $n>1$ and add to the ring $\mathcal{R}$ in Section 2 the commutativity property on the variables: $x_{i} x_{j}=x_{j} x_{i}$, for all $i, j=1,2, \ldots, n$. Observe that the rest of the 
properties of $\mathcal{R}$ still imply the existence of the matrix morphism $\sigma: \mathbb{F} \longrightarrow \mathbb{F}^{n \times n}$ and the $\sigma$-vector derivation $\delta: \mathbb{F} \longrightarrow \mathbb{F}^{n}$ by inspecting constants and variables.

Next take $a_{1}, a_{2}, \ldots, a_{n} \in \mathbb{F}$. For fixed $1 \leq i<j \leq n$, we have that

$$
\begin{gathered}
x_{j}\left(x_{i}-a_{i}\right)-x_{i}\left(x_{j}-a_{j}\right)=x_{i} a_{j}-x_{j} a_{i}=\sum_{k=1}^{n}\left(\sigma_{i, k}\left(a_{j}\right)-\sigma_{j, k}\left(a_{i}\right)\right)\left(x_{k}-a_{k}\right) \\
+\left(\sum_{k=1}^{n}\left(\sigma_{i, k}\left(a_{j}\right)-\sigma_{j, k}\left(a_{i}\right)\right) a_{k}\right)+\delta_{i}\left(a_{j}\right)-\delta_{j}\left(a_{i}\right) .
\end{gathered}
$$

Then the term (9) equals 0 for all $a_{1}, a_{2}, \ldots, a_{n} \in \mathbb{F}$ and all $0 \leq i<j \leq n$ if, and only if, $\mathbb{F}$ is commutative, $\sigma=\mathrm{Id}$ and $\delta=0$. We leave the proof to the reader.

In particular, unique remainder as in Lemma 5 can only be guaranteed in this case (variables commute and $\mathbb{F}$ is a division ring) if $\mathbb{F}$ is commutative, $\sigma=\operatorname{Id}$ and $\delta=0$. Therefore, evaluation by unique remainder division (thus "plug-in" evaluation, see Remark 10) does not exist even for multivariate conventional polynomials with commutative variables over non-commutative division rings (as considered in [1], for instance).

However, one may usually define non-trivial relations between variables while preserving evaluation properties. See Section 6 .

Remark 8. Since variables commute in many reasonable iterated skew polynomial rings, they do not satisfy the uniqueness of remainders as in Lemma 5. Thus we do not consider iterated skew polynomials in this paper, in contrast with [7]. Take for instance any iterated skew polynomial ring $\mathcal{S}=\left(\mathbb{F}\left[x_{1} ; \sigma_{1}, \delta_{1}\right]\right)\left[x_{2} ; \sigma_{2}, \delta_{2}\right]$, where $\delta_{1}=\delta_{2}=0$, $\sigma_{2}\left(x_{1}\right)=x_{1}$ and $\sigma_{1} \sigma_{2}=\sigma_{2} \sigma_{1}$. Then $x_{2} x_{1}=x_{1} x_{2}$. This can be easily extended to any number of variables. See also Remark 21.

We may now define a standard evaluation as follows, which extends the case $n=1$ from [10, 12]:

Definition 9 (Standard evaluation). For $\mathbf{a}=\left(a_{1}, a_{2}, \ldots, a_{n}\right) \in \mathbb{F}^{n}$ and a skew polynomial $F \in \mathbb{F}[\mathbf{x} ; \sigma, \delta]$, we define its $(\sigma, \delta)$-evaluation, denoted by

$$
F(\mathbf{a})=E_{\mathbf{a}}^{\sigma, \delta}(F),
$$

as the unique element $F(\mathbf{a}) \in \mathbb{F}$ such that

$$
F-F(\mathbf{a}) \in\left(x_{1}-a_{1}, x_{2}-a_{2}, \ldots, x_{n}-a_{n}\right) .
$$

We denote the corresponding total evaluation map by $E^{\sigma, \delta}$, and we use the notations $E_{\mathbf{a}}$ and $E$ when there is no confusion about $\sigma$ and $\delta$.

These evaluation maps are well-defined and left linear by Lemma 5 . To conclude, we give a recursive formula on the fundamental functions of the total evaluation map $E^{\sigma, \delta}$, which is of computational interest. This result is an extension of the case $n=1$ given in [12, Lemma 2.4] and [12, Eq. (2.3)]. 
Theorem 2. The fundamental functions $N_{\mathfrak{m}}^{\sigma, \delta}=N_{\mathfrak{m}}: \mathbb{F}^{n} \longrightarrow \mathbb{F}$, for $\mathfrak{m} \in \mathcal{M}$, of the $(\sigma, \delta)$-evaluation $E^{\sigma, \delta}$ in Definition 9 are given recursively as follows: $N_{1}(\mathbf{a})=1$, and

$$
\left(\begin{array}{c}
N_{x_{1} \mathfrak{m}}(\mathbf{a}) \\
N_{x_{2} \mathfrak{m}}(\mathbf{a}) \\
\vdots \\
N_{x_{n} \mathfrak{m}}(\mathbf{a})
\end{array}\right)=\sigma\left(N_{\mathfrak{m}}(\mathbf{a})\right) \mathbf{a}+\delta\left(N_{\mathfrak{m}}(\mathbf{a})\right)
$$

for all $\mathfrak{m} \in \mathcal{M}$ and all $\mathbf{a} \in \mathbb{F}^{n}$.

Proof. We will use the compact matrix/vector notation in (44), and we proceed recursively on $\mathfrak{m} \in \mathcal{M}$, for fixed $\mathbf{a} \in \mathbb{F}^{n}$.

Obviously, $N_{1}(\mathbf{a})=1$. Assume now that it is true for a monomial $\mathfrak{m} \in \mathcal{M}$. Therefore, there exist skew polynomials $P_{1}, P_{2}, \ldots, P_{n} \in\left(x_{1}-a_{1}, x_{2}-a_{2}, \ldots, x_{n}-a_{n}\right)$ such that, if we denote by $\mathbf{P}$ the column vector whose $i$ th row is $P_{i}$, for $i=1,2, \ldots, n$, then

$$
\begin{gathered}
\mathbf{x m}=\mathbf{P}+\mathbf{x} N_{\mathfrak{m}}(\mathbf{a})=\mathbf{P}+\sigma\left(N_{\mathfrak{m}}(\mathbf{a})\right) \mathbf{x}+\delta\left(N_{\mathfrak{m}}(\mathbf{a})\right) \\
=\mathbf{P}+\sigma\left(N_{\mathfrak{m}}(\mathbf{a})\right)(\mathbf{x}-\mathbf{a})+\left(\sigma\left(N_{\mathfrak{m}}(\mathbf{a})\right) \mathbf{a}+\delta\left(N_{\mathfrak{m}}(\mathbf{a})\right)\right),
\end{gathered}
$$

and the result follows by Lemma 5 .

Remark 10. Note that, when $\sigma=\mathrm{Id}$ and $\delta=0$, Theorem 0 states that evaluation by unique remainder coincides with evaluation performed by "plugging values" in the variables but with reversed orders (see also Remark 7). For instance, the evaluation of $x_{1} x_{2}$ at $\left(a_{1}, a_{2}\right)$ would be $a_{2} a_{1}$.

We recall that in the case $n=1$ and $\delta=0$, we have that $N_{i}(a)=N_{x^{i}}(a)=$ $\sigma^{i-1}(a) \cdots \sigma(a) a$, for $i=1,2, \ldots$, hence the notation $N_{\mathfrak{m}}$ is a reminder of its similarity with the norm function.

It has been recently shown [18] that norms as in (11) allow to naturally state Hilbert's Theorem 90 for general Galois extensions of fields (as considered by Noether) using arbitrary generators and relations of the Galois group.

\section{Conjugacy and the product rule}

From the previous section, we know that the $(\sigma, \delta)$-evaluation $E^{\sigma, \delta}$ is left linear. In this section, we will use the multiplicative properties of $\sigma, \delta$ and the fundamental functions of $E^{\sigma, \delta}$ to show that it preserves products of skew polynomials in a certain way. This property will be used in the next section to define ideals of zeros and to characterize which of them are two-sided (Proposition 18). It will be especially important in Section 8 for constructing skew polynomials of restricted degree with a given set of zeros.

We need the concept of conjugacy, where the case $n=1$ was given in [12, Eq. (2.5)].

Definition 11 (Conjugacy). Given $\mathbf{a} \in \mathbb{F}^{n}$ and $c \in \mathbb{F}^{*}$, we define the $(\sigma, \delta)$-conjugate, or just conjugate if there is no confusion, of $\mathbf{a}$ with respect to $c$ as

$$
\mathbf{a}^{c}=\sigma(c) \mathbf{a} c^{-1}+\delta(c) c^{-1} \in \mathbb{F}^{n} .
$$


We have the following properties, which extend the case $n=1$ given in [12, Eq. (2.6)].

Lemma 12. Given $\mathbf{a}, \mathbf{b} \in \mathbb{F}^{n}$ and $c, d \in \mathbb{F}^{*}$, the following properties hold:

1. $\mathbf{a}^{1}=\mathbf{a}$ and $\left(\mathbf{a}^{c}\right)^{d}=\mathbf{a}^{d c}$.

2. The relation $\mathbf{a} \sim \mathbf{b}$ if, and only if, there exist $e \in \mathbb{F}^{*}$ with $\mathbf{b}=\mathbf{a}^{e}$, is an equivalence relation on $\mathbb{F}^{n}$.

If $n=1, \sigma=\operatorname{Id}$ and $\delta=0$, then the previous notion of conjugacy coincides with the usual one on the multiplicative monoid of $\mathbb{F}$, which explains the terminology.

As noted in [13, Hilbert's Theorem 90 can be understood as any effective criterion for conjugacy, which in its classical form (cyclic Galois extensions) is given by the classical norm function. The same idea can be used to reinterpret Hilbert's Theorem 90 over any Galois extension of fields [18], where the norm function is replaced by the fundamental functions from the last section.

We may now establish and prove the product rule. The case $n=1$ was first given in [12, Th. 2.7]. We follow their proof using our matrix/vector notation.

Theorem 3 (Product rule). Given skew polynomials $F, G \in \mathbb{F}[\mathbf{x} ; \sigma, \delta]$ and $\mathbf{a} \in \mathbb{F}^{n}$, if $G(\mathbf{a})=0$, then $(F G)(\mathbf{a})=0$, and if $c=G(\mathbf{a}) \neq 0$, then

$$
(F G)(\mathbf{a})=F\left(\mathbf{a}^{c}\right) G(\mathbf{a}) .
$$

Proof. It is obvious from Lemma 5 and Definition 9 that, if $G(\mathbf{a})=0$, then $(F G)(\mathbf{a})=0$. Now assume that $c=G(\mathbf{a}) \neq 0$. First observe that

$$
\left(\mathbf{x}-\mathbf{a}^{c}\right) c=\sigma(c)(\mathbf{x}-\mathbf{a}) .
$$

Second, by Definition 9 there exist skew polynomials $P_{i}, Q_{i} \in \mathbb{F}[\mathbf{x} ; \sigma, \delta]$, for $i=$ $1,2, \ldots, n$, such that

$$
F=\mathbf{P}^{T}\left(\mathbf{x}-\mathbf{a}^{c}\right)+F\left(\mathbf{a}^{c}\right), \text { and } G=\mathbf{Q}^{T}(\mathbf{x}-\mathbf{a})+G(\mathbf{a}),
$$

where $\mathbf{P}$ and $\mathbf{Q}$ denote the column vectors whose $i$ th rows are $P_{i}$ and $Q_{i}$, respectively, for $i=1,2, \ldots, n$. Combining these facts, we obtain that

$$
\begin{gathered}
F G=F \mathbf{Q}^{T}(\mathbf{x}-\mathbf{a})+F G(\mathbf{a}) \\
=\left(F \mathbf{Q}^{T}+\mathbf{P}^{T} \sigma(c)\right)(\mathbf{x}-\mathbf{a})+F\left(\mathbf{a}^{c}\right) G(\mathbf{a}),
\end{gathered}
$$

and we are done.

This theorem can be stated when $\mathbb{F}$ is an arbitrary ring by considering only the cases where $c=0$ or $c$ is a unit. The fact that only one of these two cases happen when $\mathbb{F}$ is a division ring will be crucial in Proposition 18 and from Section 8 onwards.

Note that $\mathbf{a}^{c} \neq \mathbf{a}$ in general when $\mathbb{F}$ is non-commutative even if $\sigma=\mathrm{Id}$ and $\delta=0$. Thus the product rule is still of value for conventional polynomials over division rings. In particular, it may be that $F(\mathbf{a})=0$ and $(F G)(\mathbf{a}) \neq 0$ even for conventional polynomials, when $\mathbb{F}$ is non-commutative. 


\section{Zeros of multivariate skew polynomials}

In this section, we will define and give the basic properties of sets of zeros of multivariate skew polynomials and, conversely, sets of skew polynomials that vanish at a certain set of affine points, which will be crucial in Section 8 for Lagrange interpolation. Conceptually, they will also be important in Section 6 to define general skew polynomial rings with relations on the variables (nonfree) and where evaluation still works in a natural way.

Observe that at this point our theory loses most of its analogies with the univariate case [10, 11, 12, since $\mathbb{F}[\mathbf{x} ; \sigma, \delta]$ is not a principal ideal domain if $n>1$, hence the use of minimal skew polynomials as in [10, 11] is not possible. On the other hand, we gain analogy with respect to classical algebraic geometry:

Definition 13 (Zeros of skew polynomials). Given a set $A \subseteq \mathbb{F}[\mathbf{x} ; \sigma, \delta]$, we define its zero set as

$$
Z(A)=\left\{\mathbf{a} \in \mathbb{F}^{n} \mid F(\mathbf{a})=0, \forall F \in A\right\} .
$$

And given a set $\Omega \subseteq \mathbb{F}^{n}$, we define its associated ideal as

$$
I(\Omega)=\{F \in \mathbb{F}[\mathbf{x} ; \sigma, \delta] \mid F(\mathbf{a})=0, \forall \mathbf{a} \in \Omega\} .
$$

Observe that the ideal associated to a subset of $\mathbb{F}^{n}$ is indeed a left ideal:

Proposition 14. For any $\Omega \subseteq \mathbb{F}^{n}$, it holds that $I(\Omega) \subseteq \mathbb{F}[\mathbf{x} ; \sigma, \delta]$ is a left ideal.

Proof. It follows directly from the product rule (Theorem 3). Alternatively, it can be proven by noting that $I(\Omega)=\bigcap_{\mathbf{a} \in \Omega}\left(x_{1}-a_{1}, x_{2}-a_{2}, \ldots, x_{n}-a_{n}\right)$.

We next list some basic properties of zero sets and ideals of zeros that follow from the definitions, in the same way as in classical algebraic geometry.

Proposition 15. Let $\Omega, \Omega_{1}, \Omega_{2} \subseteq \mathbb{F}^{n}$ and $A, A_{1}, A_{2} \subseteq \mathbb{F}[\mathbf{x} ; \sigma, \delta]$ be arbitrary sets. The following properties hold:

1. $I(\{\mathbf{a}\})=\left(x_{1}-a_{1}, x_{2}-a_{2}, \ldots, x_{n}-a_{n}\right)$ and $Z\left(x_{1}-a_{1}, x_{2}-a_{2}, \ldots, x_{n}-a_{n}\right)=\{\mathbf{a}\}$, for all $\mathbf{a}=\left(a_{1}, a_{2}, \ldots, a_{n}\right) \in \mathbb{F}^{n}$.

2. $I(\varnothing)=(1)$ and $Z(1)=\varnothing$.

3. $I\left(\mathbb{F}^{n}\right) \subseteq I(\Omega)$ and $Z(\{0\})=\mathbb{F}^{n}$. That is, $I\left(\mathbb{F}^{n}\right)$ is the minimal ideal of zeros.

4. If $\Omega_{1} \subseteq \Omega_{2}$, then $I\left(\Omega_{2}\right) \subseteq I\left(\Omega_{1}\right)$.

5. If $A_{1} \subseteq A_{2}$, then $Z\left(A_{2}\right) \subseteq Z\left(A_{1}\right)$.

6. $I\left(\Omega_{1} \cup \Omega_{2}\right)=I\left(\Omega_{1}\right) \cap I\left(\Omega_{2}\right)$.

7. $Z(A)=Z((A))$ and $Z\left(A_{1} \cup A_{2}\right)=Z\left(\left(A_{1}\right)+\left(A_{2}\right)\right)=Z\left(A_{1}\right) \cap Z\left(A_{2}\right)$.

8. $\Omega \subseteq Z(I(\Omega))$, and equality holds if, and only if, $\Omega=Z(B)$ for some $B \subseteq \mathbb{F}[\mathbf{x} ; \sigma, \delta]$. 
9. $A \subseteq(A) \subseteq I(Z(A))$, and equality holds if, and only if, $A=I(\Psi)$ for some $\Psi \subseteq \mathbb{F}^{n}$.

Item 8 in the previous proposition motivates the definition of P-closed sets, where the case $n=1$ was given in [10, 12]:

Definition 16 (P-closures). Given a subset $\Omega \subseteq \mathbb{F}^{n}$, we define its P-closure as

$$
\bar{\Omega}=Z(I(\Omega)),
$$

and we say that $\Omega$ is P-closed if $\bar{\Omega}=\Omega$.

By Proposition 15, Item 8, P-closed sets correspond to sets of zeros of sets of skew polynomials, and we have the following:

Lemma 17. Given a subset $\Omega \subseteq \mathbb{F}^{n}$, it holds that $\bar{\Omega}$ is the smallest P-closed subset of $\mathbb{F}^{n}$ containing $\Omega$.

\section{General and minimal skew polynomial rings}

In this section, we define general skew polynomial rings as those with a set of relations on the variables and where evaluation is still as in Definition9. In particular, by considering a maximum set of such relations, we may define minimal skew polynomial rings.

Note that the whole space $\mathbb{F}^{n}$ is P-closed, and Item 3 in Proposition 15 says that, for evaluation purposes, we may just consider the quotient left module

$$
\mathbb{F}[\mathbf{x} ; \sigma, \delta] / I\left(\mathbb{F}^{n}\right),
$$

which is a ring if $I\left(\mathbb{F}^{n}\right)$ is a two-sided ideal, and in such a case we obtain the above mentioned minimal skew polynomial ring where the $(\sigma, \delta)$-standard evaluation is still defined.

In the following proposition, we characterize when an ideal of zeros is two-sided, which includes in particular the ideal $I\left(\mathbb{F}^{n}\right)$ :

Proposition 18. Given a subset $\Omega \subseteq \mathbb{F}^{n}$, the following are equivalent:

1. $I(\Omega)$ is a two-sided ideal.

2. If $F \in I(\Omega)$ and $c \in \mathbb{F}$, then $F c \in I(\Omega)$.

3. If $\mathbf{a} \in \bar{\Omega}$, then $\mathbf{a}^{c} \in \bar{\Omega}$, for all $c \in \mathbb{F}^{*}$.

4. If $\mathbf{a} \in \Omega$, then $\mathbf{a}^{c} \in \bar{\Omega}$, for all $c \in \mathbb{F}^{*}$.

In particular, $I\left(\mathbb{F}^{n}\right)$ is a two-sided ideal. 
Proof. We prove the following implications:

1) $\Longrightarrow 2$ ): Trivial.

$2) \Longrightarrow 3)$ : Let $\mathbf{a} \in \bar{\Omega}, F \in I(\Omega)$ and $c \in \mathbb{F}^{*}$. First, it holds that $I(\Omega)=I(\bar{\Omega})$ by Items 8 and 9 in Proposition 15, and $F c \in I(\Omega)$ by hypothesis. Thus

$$
0=(F c)(\mathbf{a})=F\left(\mathbf{a}^{c}\right) c
$$

by the product rule (Theorem 3 ). Hence $\mathbf{a}^{c} \in Z(I(\Omega))=\bar{\Omega}$.

$3) \Longrightarrow 4$ ): Trivial from $\Omega \subseteq \bar{\Omega}$.

4) $\Longrightarrow 1)$ : Let $F \in I(\Omega)$ and $G \in \mathbb{F}[\mathbf{x} ; \sigma, \delta]$, fix $\mathbf{a} \in \Omega$ and define $c=G(\mathbf{a})$. If $c=0$, then $(F G)(\mathbf{a})=0$ by the product rule. If $c \neq 0$, by hypothesis and the product rule, we have that

$$
(F G)(\mathbf{a})=F\left(\mathbf{a}^{c}\right) G(\mathbf{a})=0,
$$

since $\mathbf{a}^{c} \in \bar{\Omega}$ and $F \in I(\Omega)=I(\bar{\Omega})$. Hence $(F G)(\mathbf{a})=0$ for any $\mathbf{a} \in \Omega$ and thus $F G \in I(\Omega)$, and we are done.

Observe that, to prove 4$) \Longrightarrow 1$ ), we use that $\mathbb{F}$ is a division ring, since we use that every $c \in \mathbb{F} \backslash\{0\}$ is invertible.

We may now define (nonfree) general skew polynomial rings and, in particular, a minimal one.

Definition 19 (Skew polynomial rings). For any two-sided ideal $I \subseteq I\left(\mathbb{F}^{n}\right)$, we say that the quotient ring

$$
\mathbb{F}[\mathbf{x} ; \sigma, \delta] / I
$$

is a skew polynomial ring with matrix morphism $\sigma$ and vector derivation $\delta$. The minimal skew polynomial ring with matrix morphism $\sigma$ and vector derivation $\delta$ is defined as that obtained when $I=I\left(\mathbb{F}^{n}\right)$.

This is exactly what happens with multivariate conventional polynomial rings (the case $\sigma=\operatorname{Id}$ and $\delta=0$ ) over fields. One may consider the free multivariate polynomial ring and define the conventional evaluation on it, either by plugging values in the variables or equivalently by unique remainder division. When $\mathbb{F}$ is a field, $I\left(\mathbb{F}^{n}\right)$ contains the two-sided ideal $J$ generated by $x_{i} x_{j}-x_{j} x_{i}$, for $1 \leq i<j \leq n$. If $\mathbb{F}$ is infinite, then $J=I\left(\mathbb{F}^{n}\right)$, whereas

$$
I\left(\mathbb{F}^{n}\right)=J+\left(x_{1}^{q}-x_{1}, x_{2}^{q}-x_{2}, \ldots, x_{n}^{q}-x_{n}\right)
$$

if $\mathbb{F}$ is finite and has $q$ elements. The results in the following sections will be proven for the free multivariate skew polynomial ring. By projecting onto the quotient, they can also be stated for any multivariate skew polynomial ring.

We conclude this section by showing that skew polynomial rings can form iterated sequences of rings by adding variables, even though we do not consider iterated skew polynomial rings in the standard way, as shown in Remark 8 . The proof of the following result is straightforward. 
Proposition 20. Let $0<r<n$ be a positive integer, let $\tau: \mathbb{F} \longrightarrow \mathbb{F}^{r \times r}$ and $\nu: \mathbb{F} \longrightarrow$ $\mathbb{F}^{(n-r) \times(n-r)}$ be matrix morphisms and let $\delta_{\tau}: \mathbb{F} \longrightarrow \mathbb{F}^{r}$ and $\delta_{\nu}: \mathbb{F} \longrightarrow \mathbb{F}^{(n-r)}$ be a $\tau$-vector derivation and a $\nu$-vector derivation, respectively. Define $\sigma: \mathbb{F} \longrightarrow \mathbb{F}^{n \times n}$ and $\delta_{\sigma}: \mathbb{F} \longrightarrow \mathbb{F}^{n}$ by

$$
\sigma(a)=\left(\begin{array}{cc}
\tau(a) & 0 \\
0 & \nu(a)
\end{array}\right) \quad \text { and } \quad \delta_{\sigma}(a)=\left(\begin{array}{c}
\delta_{\tau}(a) \\
\delta_{\nu}(a)
\end{array}\right)
$$

for all $a \in \mathbb{F}$. Then $\sigma$ is a matrix morphism and $\delta_{\sigma}$ is a $\sigma$-vector derivation. Consider now the natural inclusion map

$$
\rho: \mathbb{F}\left[x_{1}, x_{2}, \ldots, x_{r} ; \tau, \delta_{\tau}\right] \longrightarrow \mathbb{F}\left[x_{1}, x_{2}, \ldots, x_{n} ; \sigma, \delta_{\sigma}\right] .
$$

The following properties hold:

1. $\rho$ is a one to one ring morphism.

2. For all $F \in \mathbb{F}\left[x_{1}, x_{2}, \ldots, x_{r} ; \tau, \delta_{\tau}\right]$, all $\mathbf{a}_{\tau} \in \mathbb{F}^{r}$ and all $\mathbf{a}_{\nu} \in \mathbb{F}^{n-r}$, it holds that

$$
E_{\mathbf{a}_{\tau}}^{\tau, \delta_{\tau}}(F)=E_{\mathbf{a}_{\sigma}}^{\sigma, \delta_{\sigma}}(\rho(F)),
$$

where $\mathbf{a}_{\sigma}=\left(\mathbf{a}_{\tau}, \mathbf{a}_{\nu}\right) \in \mathbb{F}^{n}$.

3. For any two-sided ideal $J \subseteq I\left(\mathbb{F}^{n}\right)$, it holds that $\rho^{-1}(J) \subseteq I\left(\mathbb{F}^{r}\right)$ is a two-sided ideal and $\rho$ can be restricted to a one to one ring morphism

$$
\rho: \mathbb{F}\left[x_{1}, x_{2}, \ldots, x_{r} ; \tau, \delta_{\tau}\right] / \rho^{-1}(J) \longrightarrow \mathbb{F}\left[x_{1}, x_{2}, \ldots, x_{n} ; \sigma, \delta_{\sigma}\right] / J .
$$

This holds in particular choosing $J=I\left(\mathbb{F}^{n}\right)$, which implies that $\rho^{-1}(J)=I\left(\mathbb{F}^{r}\right)$.

In particular, if $\sigma$ and $\delta$ are given as in Example 3, then $\mathbb{F}[\mathbf{x} ; \sigma, \delta]$ contains a sequence of $n$ nested skew polynomial rings, where the first one is the univariate skew polynomial ring $\mathbb{F}\left[x_{1} ; \sigma_{1}, \delta_{1}\right]$.

Remark 21. Iterated skew polynomial rings such that $\delta_{i}(\mathbb{F}) \subseteq \mathbb{F}+\mathbb{F} x_{1}+\cdots+\mathbb{F} x_{i-1}$, for $i=1,2, \ldots, n$, are also quotients of free multivariate skew polynomial rings since they satisfy the rules (11), setting $\sigma_{i, i}(a)=\sigma_{i}(a)$ and $\sigma_{i, j}(a)$ as the coefficient of $x_{j}$ in $\delta_{i}(a)$ for $j<i$ (note that necessarily $\sigma_{i}(\mathbb{F}) \subseteq \mathbb{F}$, for $i=1,2, \ldots, n$ ). Use for instance the universal

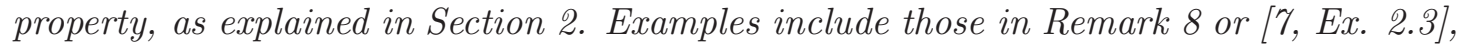
and important rings such as Weyl algebras [6] or solvable iterated skew polynomial rings [9, 23]. In particular, when evaluation can be given for such iterated skew polynomials by unique remainder as in [7], it must coincide with our notion of evaluation (Definition 9). What happens is that these iterated skew polynomial rings are in general quotients by a two-sided ideal $J$ that satisfies that $J \backslash I\left(\mathbb{F}^{n}\right) \neq \varnothing$ and $I\left(\mathbb{F}^{n}\right) \backslash J \neq \varnothing$. 


\section{$7 \quad$ P-generators, P-independence and P-bases}

The main feature of P-closed sets is that they can be "generated" by certain subsets, called $P$-bases, that control the possible values given by a function defined by a skew polynomial on such sets, as we will show in the next section. P-bases are given by a P-independence notion and naturally form a matroid (Proposition 27). P-independence was defined for the case $n=1$ in [10, 11]. We start with the main definitions:

Definition 22 (P-generators). Given a P-closed set $\Omega \subseteq \mathbb{F}^{n}$, we say that $\mathcal{G} \subseteq \Omega$ generates $\Omega$ if $\overline{\mathcal{G}}=\Omega$, and it is then called a set of P-generators for $\Omega$. We say that $\Omega$ is finitely generated if it has a finite set of $\mathrm{P}$-generators.

Definition 23 (P-independence). We say that $\mathbf{a} \in \mathbb{F}^{n}$ is P-independent from $\Omega \subseteq \mathbb{F}^{n}$ if it does not belong to $\bar{\Omega}$.

A set $\Omega \subseteq \mathbb{F}^{n}$ is called P-independent if every $\mathbf{a} \in \Omega$ is P-independent from $\Omega \backslash\{\mathbf{a}\}$. P-dependent means not P-independent.

Definition 24 (P-bases). Given a P-closed set $\Omega \subseteq \mathbb{F}^{n}$, we say that a subset $\mathcal{B} \subseteq \Omega$ is a P-basis of $\Omega$ if it is P-independent and $\overline{\mathcal{B}}=\Omega$.

The following is the main result of this section, where Item 3 will be crucial in order to perform Lagrange interpolation recursively.

Proposition 25. Given sets $\mathcal{B} \subseteq \Omega \subseteq \mathbb{F}^{n}$, where $\Omega=\overline{\mathcal{B}}$, the following are equivalent:

1. $\mathcal{B}$ is a P-basis of $\Omega$.

2. If $\mathcal{G} \subseteq \mathcal{B}$ and $\overline{\mathcal{G}}=\Omega$, then $\mathcal{G}=\mathcal{B}$. That is, $\mathcal{B}$ is a minimal set of P-generators of $\Omega$.

3. (If $\mathcal{B}$ is finite) For any ordering $\mathbf{b}_{1}, \mathbf{b}_{2}, \ldots, \mathbf{b}_{M}$ of the elements in $\mathcal{B}$ and for $i=$ $0,1,2, \ldots, M-1$, it holds that $\mathbf{b}_{i+1}$ is P-independent from $\mathcal{B}_{i}=\left\{\mathbf{b}_{1}, \mathbf{b}_{2}, \ldots, \mathbf{b}_{i}\right\}$, where $\mathcal{B}_{0}=\varnothing$.

Proof. We prove each implication separately:

$1) \Longrightarrow 2$ ): Assume that there exists $\mathcal{G} \varsubsetneqq \mathcal{B}$ with $\overline{\mathcal{G}}=\Omega$ and let $\mathbf{a} \in \mathcal{B} \backslash \mathcal{G}$. Then

$$
\mathbf{a} \in \Omega=\overline{\mathcal{G}}=\overline{\mathcal{B} \backslash\{\mathbf{a}\}},
$$

hence Item 1 does not hold.

$2) \Longrightarrow 1$ ): Assume that $\mathcal{B}$ is not $\mathrm{P}$-independent and take $\mathbf{a} \in \mathcal{B}$ with $\mathbf{a} \in \overline{\mathcal{B} \backslash\{\mathbf{a}\}}$. Define $\mathcal{G}=\mathcal{B} \backslash\{\mathbf{a}\} \varsubsetneqq \mathcal{B}$. It holds that

$$
\mathcal{B}=\{\mathbf{a}\} \cup(\mathcal{B} \backslash\{\mathbf{a}\}) \subseteq \overline{\mathcal{G}},
$$

hence $\overline{\mathcal{G}}=\Omega$ and Item 2 does not hold.

$1) \Longrightarrow 3$ ): Assume that $\mathbf{b}_{i+1}$ is $\mathrm{P}$-dependent from $\mathcal{B}_{i}$ for a given $i$ and a given ordering of $\mathcal{B}$. Then

$$
\mathbf{b}_{i+1} \in \overline{\mathcal{B}_{i}} \subseteq \overline{\mathcal{B} \backslash\left\{\mathbf{b}_{i+1}\right\}},
$$


hence Item 1 does not hold.

$3) \Longrightarrow 1$ ): Assume that $\mathbf{a}$ is $\mathrm{P}$-dependent from $\mathcal{B} \backslash\{\mathbf{a}\}$ and order the $M$ elements in $\mathcal{B}$ in such a way that $\mathbf{b}_{M}=\mathbf{a}$. Then $\mathbf{b}_{M}$ is P-dependent from $\mathcal{B}_{M-1}$ and Item 3 does not hold.

We have the following important immediate consequence of Item 2 in the previous proposition:

Corollary 26. If a P-closed set is finitely generated, then it admits a finite P-basis.

Finally, we observe that the family of P-independent sets forms a matroid [21, Sec. 1.1] whose bases [21, Sec. 1.2] are precisely the family of P-bases. The proof requires results from the following sections, but we will state the observation in this section for clarity.

Proposition 27. For every finitely generated P-closed set $\Omega \subseteq \mathbb{F}^{n}$, the pair $\left(\mathcal{P}(\Omega), \mathcal{I}_{\Omega}\right)$ forms a matroid, where $\mathcal{P}(\Omega)$ is the collection of all subsets of $\Omega$, and $\mathcal{I}_{\Omega}$ is the collection of P-independent subsets of $\Omega$. Furthermore, the bases of the matroid $\left(\mathcal{P}(\Omega), \mathcal{I}_{\Omega}\right)$ are precisely the $P$-bases of $\Omega$.

Proof. First, it is trivial to see that $\varnothing \in \mathcal{I}_{\Omega}$ and, if $\mathcal{A}^{\prime} \subseteq \mathcal{A}$ and $\mathcal{A} \in \mathcal{I}_{\Omega}$, then $\mathcal{A}^{\prime} \in \mathcal{I}_{\Omega}$. The augmentation property of matroids is the first statement in Lemma 36, proven in Section 9. Finally, the fact that bases (that is, maximal independent sets) and P-bases coincide is the second statement in Lemma 36 .

\section{Skew polynomial functions and Lagrange interpolation}

In this section, we give the main result of this paper. We show, in a Lagrange-type interpolation theorem, what values a function given by a skew polynomial can take when evaluated on a finitely generated P-closed set (Theorem 4). This result will be crucial in the following sections to describe the image and kernel of the evaluation map defined below (Theorem 51), to prove later that P-independent sets form a matroid (Lemma 36) and that the rank of a P-closed set is the rank of the corresponding skew Vandermonde matrix (Proposition 41). On the way, we derive other important results on P-closed sets.

Observe first that the total $(\sigma, \delta)$-evaluation gives a left linear map

$$
E_{\Omega}^{\sigma, \delta}: \mathbb{F}[\mathbf{x} ; \sigma, \delta] \longrightarrow \mathbb{F}^{\Omega},
$$

when restricted to evaluating over a subset $\Omega \subseteq \mathbb{F}^{n}$ or, in other words, by composing $E_{\Omega}^{\sigma, \delta}=\pi_{\Omega} \circ E^{\sigma, \delta}$, where $\pi_{\Omega}: \mathbb{F}^{\mathbb{F}^{n}} \longrightarrow \mathbb{F}^{\Omega}$ is the canonical projection map.

Hence $E_{\Omega}^{\sigma, \delta}$ gives a correspondence between multivariate skew polynomials $F \in$ $\mathbb{F}[\mathbf{x} ; \sigma, \delta]$ and some particular functions $f=E_{\Omega}^{\sigma, \delta}(F): \Omega \longrightarrow \mathbb{F}$. Such functions will be called multivariate skew polynomial functions over $\Omega$.

Formally, the objective of this section and the next one is to describe the kernel and image of the map $E_{\Omega}^{\sigma, \delta}$ when $\Omega$ is P-closed and finitely generated. We start with the following lemma, which is a key tool in Lagrange interpolation: 
Lemma 28. Let $\mathcal{B} \subseteq \mathbb{F}^{n}$ be a finite P-independent set and let $\mathbf{b} \notin \overline{\mathcal{B}}$. There exists $F \in I(\mathcal{B}) \backslash I(\mathcal{B} \cup\{\mathbf{b}\})$ such that $\operatorname{deg}(F) \leq \# \mathcal{B}$.

Proof. First we prove that $I(\mathcal{B}) \backslash I(\mathcal{B} \cup\{\mathbf{b}\}) \neq \varnothing$. Assume the opposite. Then

$$
\overline{\mathcal{B}}=Z(I(\mathcal{B}))=Z(I(\mathcal{B} \cup\{\mathbf{b}\})),
$$

and $\mathcal{B} \cup\{\mathbf{b}\} \subseteq Z(I(\mathcal{B} \cup\{\mathbf{b}\}))$ by Item 8 in Proposition 15, Thus $\mathbf{b} \in \overline{\mathcal{B}}$, which is a contradiction.

Now let $\mathcal{B}=\left\{\mathbf{b}_{1}, \mathbf{b}_{2}, \ldots, \mathbf{b}_{M}\right\}$ with $M=\# \mathcal{B}$, let $\prec$ be any ordering of $\mathcal{M}$ preserving degrees, and take $F \in I(\mathcal{B}) \backslash I(\mathcal{B} \cup\{\mathbf{b}\})$ such that $\operatorname{LM}(F)$ is minimum possible with respect to $\prec$. Assume that $\operatorname{deg}(F) \geq M+1$, which implies that $\operatorname{deg}(\operatorname{LM}(F)) \geq M+1$ by the choice of the ordering $\prec$.

Let $\operatorname{LM}(F)=\mathfrak{m} x_{i_{1}} x_{i_{2}} \cdots x_{i_{M+1}}$, for some $\mathfrak{m} \in \mathcal{M}$. By the product rule (Theorem 3), we may choose elements $a_{1}, a_{2}, \ldots, a_{M+1} \in \mathbb{F}$ such that

$$
G=\mathfrak{m}\left(x_{i_{1}}-a_{1}\right)\left(x_{i_{2}}-a_{2}\right) \cdots\left(x_{i_{M+1}}-a_{M+1}\right)
$$

satisfies that $G\left(\mathbf{b}_{i}\right)=0$, for $i=1,2, \ldots, M+1$, denoting $\mathbf{b}_{M+1}=\mathbf{b}$. In particular, there exists $a \in \mathbb{F}$ such that $H=F-a G$ satisfies $\operatorname{LM}(H) \prec \operatorname{LM}(F)$, since $\operatorname{LM}(F)=\operatorname{LM}(G)$. Now, by the definition of $G$, it holds that

$$
H=F-a G \in I(\mathcal{B}) \backslash I(\mathcal{B} \cup\{\mathbf{b}\}),
$$

which is absurd by the minimality of $\operatorname{LM}(F)$. Therefore $\operatorname{deg}(F) \leq M$ and we are done.

Remark 29. Note that, to construct $G$ in the previous proof, we are implicitly using that $\mathbb{F}$ is a division ring and we are implicitly applying Theorem 0 in its full form. Note however that constructing $G$ is only needed to ensure the bound $\operatorname{deg}(F) \leq \# \mathcal{B}$, but the existence of $F$ still holds if $\mathbb{F}$ is an arbitrary ring. The bound on $\operatorname{deg}(F)$ will only be used to define skew Vandermonde matrices in Section 10. We do not investigate the full validity of Lemma 28 when $\mathbb{F}$ is an arbitrary ring.

The main result of this section is a Lagrange-type interpolation theorem in $\mathbb{F}[\mathbf{x} ; \sigma, \delta]$, whose proof is given by an iterative Newton-type algorithm thanks to Item 3 in Proposition 25. This result extends the case $n=1$ given in [10, Th. 8] (see also the beginning of [12, Sec. 5]). Newton-type iterative algorithms have been given in [22] for univariate skew polynomials, and in [16] for their free left modules.

Theorem 4 (Lagrange interpolation). Let $\Omega \subseteq \mathbb{F}^{n}$ be a finitely generated P-closed set with finite P-basis $\mathcal{B}=\left\{\mathbf{b}_{1}, \mathbf{b}_{2}, \ldots, \mathbf{b}_{M}\right\}$. The following hold:

1. If $E_{\mathcal{B}}^{\sigma, \delta}(F)=E_{\mathcal{B}}^{\sigma, \delta}(G)$, then $E_{\Omega}^{\sigma, \delta}(F)=E_{\Omega}^{\sigma, \delta}(G)$, for all $F, G \in \mathbb{F}[\mathbf{x} ; \sigma, \delta]$. That is, the values of a skew polynomial function $f: \Omega \longrightarrow \mathbb{F}$ are uniquely given by $f\left(\mathbf{b}_{1}\right), f\left(\mathbf{b}_{2}\right), \ldots, f\left(\mathbf{b}_{M}\right)$. 
2. For every $a_{1}, a_{2}, \ldots, a_{M} \in \mathbb{F}$, there exists $F \in \mathbb{F}[\mathbf{x} ; \sigma, \delta]$ such that $\operatorname{deg}(F)<M$ and $F\left(\mathbf{b}_{i}\right)=a_{i}$, for $i=1,2, \ldots, M$.

Proof. We prove each item separately.

1. We just need to prove that $E_{\mathcal{B}}^{\sigma, \delta}(F)=0$ implies $E_{\Omega}^{\sigma, \delta}(F)=0$. By definition, $\mathcal{B} \subseteq Z(F)$, and by Proposition 15 , it holds that $I(Z(F)) \subseteq I(\mathcal{B})$ and

$$
\Omega=\overline{\mathcal{B}}=Z(I(\mathcal{B})) \subseteq Z(I(Z(F)))=Z(F),
$$

and the result follows.

2. Let $\mathcal{B}=\left\{\mathbf{b}_{1}, \mathbf{b}_{2}, \ldots, \mathbf{b}_{M}\right\}$ as in Proposition 25, Item 3. We prove the result iteratively for each of the P-independent sets $\mathcal{B}_{i}, i=1,2, \ldots, M$, as in Newton's algorithm.

We start by defining the skew polynomial $F_{1}=a_{1}$, which obviously satisfies $F_{1}\left(\mathbf{b}_{1}\right)=a_{1}$ and $\operatorname{deg}\left(F_{1}\right)<1$. Now assume that $M>1,1 \leq i \leq M-1$ and there exists a skew polynomial $F_{i}$ such that $F_{i}\left(\mathbf{b}_{j}\right)=a_{j}$, for $j=1,2, \ldots, i$, and $\operatorname{deg}\left(F_{i}\right)<i$. By Lemma 28, there exists

$$
G \in I\left(\left\{\mathbf{b}_{1}, \mathbf{b}_{2}, \ldots, \mathbf{b}_{i}\right\}\right) \backslash I\left(\left\{\mathbf{b}_{1}, \mathbf{b}_{2}, \ldots, \mathbf{b}_{i+1}\right\}\right)
$$

such that $\operatorname{deg}(G)<i+1$. The skew polynomial

$$
F_{i+1}=F_{i}+\left(a_{i+1}-F_{i}\left(\mathbf{b}_{i+1}\right)\right) G\left(\mathbf{b}_{i+1}\right)^{-1} G
$$

satisfies that $F_{i+1}\left(\mathbf{b}_{j}\right)=a_{j}$, for $j=1,2, \ldots, i+1$, and $\operatorname{deg}\left(F_{i+1}\right)<i+1$.

In the rest of the section, we derive some important consequences of this theorem. We start with the concept of dual P-bases.

Definition 30 (Dual P-bases). Given a finite P-basis $\mathcal{B}=\left\{\mathbf{b}_{1}, \mathbf{b}_{2}, \ldots, \mathbf{b}_{M}\right\}$ of a Pclosed set $\Omega \subseteq \mathbb{F}^{n}$, we say that a set of skew polynomials

$$
\mathcal{B}^{*}=\left\{F_{1}, F_{2}, \ldots, F_{M}\right\} \subseteq \mathbb{F}[\mathbf{x} ; \sigma, \delta]
$$

is a dual P-basis of $\mathcal{B}$ if $F_{i}\left(\mathbf{b}_{j}\right)=\delta_{i, j}$ for all $i, j=1,2, \ldots, M$.

We have the following immediate consequence of Theorem 4 on the existence and uniqueness of dual P-bases:

Corollary 31. Any finite P-basis, with $M$ elements, of a P-closed set $\Omega$ admits a dual $P$-basis consisting of $M$ skew polynomials of degree less than $M$. Moreover, any two dual P-bases of the same P-basis define the same skew polynomial functions over $\Omega$.

An important consequence of Theorem 4 is the following result on the sizes of P-bases: 
Corollary 32. Any two P-bases of a finitely generated P-closed set are finite and have the same number of elements.

Proof. Given a finite P-basis $\mathcal{B}=\left\{\mathbf{b}_{1}, \mathbf{b}_{2}, \ldots, \mathbf{b}_{M}\right\}$ of size $M$ of a P-closed set $\Omega$, we will show first that $\operatorname{Im}\left(E_{\Omega}^{\sigma, \delta}\right)$ is a left vector subspace of $\mathbb{F}^{\Omega}$ with basis

$$
\left\{E_{\Omega}^{\sigma, \delta}\left(F_{1}\right), E_{\Omega}^{\sigma, \delta}\left(F_{2}\right), \ldots, E_{\Omega}^{\sigma, \delta}\left(F_{M}\right)\right\}
$$

for any dual P-basis $\left\{F_{1}, F_{2}, \ldots, F_{M}\right\}$ of $\mathcal{B}$. Assume that there exist $\lambda_{1}, \lambda_{2}, \ldots, \lambda_{M} \in \mathbb{F}$ such that $\sum_{i=1}^{M} \lambda_{i} E_{\Omega}^{\sigma, \delta}\left(F_{i}\right)=0$. Defining $F=\sum_{i=1}^{M} \lambda_{i} F_{i}$, it follows that

$$
E_{\Omega}^{\sigma, \delta}(F)=E_{\Omega}^{\sigma, \delta}\left(\sum_{i=1}^{M} \lambda_{i} F_{i}\right)=\sum_{i=1}^{M} \lambda_{i} E_{\Omega}^{\sigma, \delta}\left(F_{i}\right)=0,
$$

thus $\lambda_{i}=F\left(\mathbf{b}_{i}\right)=0$, for $i=1,2, \ldots, M$, and the set in (16) is left linearly independent. Now, given $F \in \mathbb{F}[\mathbf{x} ; \sigma, \delta]$, define $G=F-\sum_{i=1}^{M} F\left(\mathbf{b}_{i}\right) F_{i}$. By definition, we have that $E_{\mathcal{B}}^{\sigma, \delta}(G)=0$. Therefore $E_{\Omega}^{\sigma, \delta}(G)=0$ by Theorem 4 , thus

$$
E_{\Omega}^{\sigma, \delta}(F)=\sum_{i=1}^{M} F\left(\mathbf{b}_{i}\right) E_{\Omega}^{\sigma, \delta}\left(F_{i}\right),
$$

and we conclude that the set in (16) is a left basis of $\operatorname{Im}\left(E_{\Omega}^{\sigma, \delta}\right)$.

In particular, $\operatorname{dim}\left(\operatorname{Im}\left(E_{\Omega}^{\sigma, \delta}\right)\right)=M$ and the result follows for finite P-bases, since $\operatorname{dim}\left(\operatorname{Im}\left(E_{\Omega}^{\sigma, \delta}\right)\right)$ is independent of the choice of finite P-basis (since $\mathbb{F}$ is a division ring).

Finally, if there exists an infinite P-basis $\mathcal{B}^{\prime}$ of $\Omega$, we may take a P-independent subset $\mathcal{C} \subseteq \mathcal{B}^{\prime}$ of size $M+1$, define $\Psi=\overline{\mathcal{C}} \subseteq \Omega$ and we would have that $\operatorname{dim}\left(\operatorname{Im}\left(E_{\Psi}^{\sigma, \delta}\right)\right)=M+1$, and the canonical projection map

$$
\pi_{\Psi}: \operatorname{Im}\left(E_{\Omega}^{\sigma, \delta}\right) \longrightarrow \operatorname{Im}\left(E_{\Psi}^{\sigma, \delta}\right)
$$

is onto. This is absurd (since $\mathbb{F}$ is a division ring) and the result follows.

We conclude with the following natural definition, which is motivated by the previous corollary. It is an extension of the case $n=1$ given in [10, 12].

Definition 33 (Rank of P-closed sets). Given a finitely generated P-closed set $\Omega \subseteq$ $\mathbb{F}^{n}$, we define its rank, denoted by $\operatorname{Rk}(\Omega)$, as the size of any of its $\mathrm{P}$-bases.

Remark 34. If $\Omega$ is a finitely generated P-closed set, then $\operatorname{Rk}(\Omega)$ coincides with the rank of the matroid $\left(\mathcal{P}(\Omega), \mathcal{I}_{\Omega}\right)$ from Proposition 27 (see [21, Sec. 1.3]). Note that we make use of Corollary 32 to prove later in Lemma 36 that $\left(\mathcal{P}(\Omega), \mathcal{I}_{\Omega}\right)$ is indeed a matroid. 


\section{The image and kernel of the evaluation map}

In this section, we describe the left vector space of skew polynomial functions and, to that end, we obtain the dimensions and some left bases of the image and kernel of the evaluation map (Theorem 5). As a conclusion to the section, we also deduce a finitedimensional left vector space description of quotients of a skew polynomial ring, which includes the minimal skew polynomial ring when $\mathbb{F}$ is a finite field.

We need the following auxiliary lemmas. The first can be seen as a refinement of Item 1 in Theorem 4:

Lemma 35. Let $\Omega \subseteq \mathbb{F}^{n}$ be a finitely generated P-closed set and let $\mathcal{G} \subseteq \Omega$. It holds that $\Omega=\overline{\mathcal{G}}$ if, and only if,

$$
\operatorname{dim}\left(\operatorname{Im}\left(E_{\Omega}^{\sigma, \delta}\right)\right)=\operatorname{dim}\left(\operatorname{Im}\left(E_{\mathcal{G}}^{\sigma, \delta}\right)\right) .
$$

Proof. First, recall that the given dimensions are finite due to the proof of Corollary 32 The direct implication is in essence Item 1 in Theorem 4. For the reversed implication, the equality on dimensions implies that the projection map $\operatorname{Im}\left(E_{\Omega}^{\sigma, \delta}\right) \longrightarrow \operatorname{Im}\left(E_{\mathcal{G}}^{\sigma, \delta}\right)$ is a left vector space isomorphism. Thus $I(\mathcal{G})=I(\Omega)$, which implies that

$$
\overline{\mathcal{G}}=Z(I(\mathcal{G}))=Z(I(\Omega))=\Omega .
$$

The next lemma is a further refinement of Proposition 25.

Lemma 36. If $\mathcal{B} \subseteq \mathbb{F}^{n}$ is finite and P-independent, and $\mathbf{a} \in \mathbb{F}^{n} \backslash \overline{\mathcal{B}}$, then $\mathcal{B}^{\prime}=\mathcal{B} \cup\{\mathbf{a}\}$ is P-independent.

As a consequence, a finite subset $\mathcal{B} \subseteq \mathbb{F}^{n}$ is a P-basis of a finitely generated $P$-closed set $\mathcal{B} \subseteq \Omega \subseteq \mathbb{F}^{n}$ if, and only if, the following property holds: $\mathcal{B}$ is P-independent, and if $\mathcal{B} \subseteq \mathcal{G} \subseteq \Omega$ and $\mathcal{G}$ is $P$-independent, then $\mathcal{G}=\mathcal{B}$. That is, $\mathcal{B}$ is a maximal $P$-independent set in $\Omega$.

Proof. Since $\mathbf{a} \notin \overline{\mathcal{B}}$, it holds that $I(\mathcal{B}) \backslash I\left(\mathcal{B}^{\prime}\right) \neq \varnothing$, as in the proof of Lemma 28. Thus

$$
\operatorname{dim}\left(\operatorname{Im}\left(E_{\mathcal{B}^{\prime}}^{\sigma, \delta}\right)\right) \geq \operatorname{dim}\left(\operatorname{Im}\left(E_{\mathcal{B}}^{\sigma, \delta}\right)\right)+1 .
$$

By the previous lemma and the proof of Corollary [32, we conclude that $\operatorname{Rk}\left(\overline{\mathcal{B}^{\prime}}\right)=$ $\operatorname{Rk}(\overline{\mathcal{B}})+1$. Again by Corollary 32 and its proof, we conclude that $\mathcal{B}^{\prime}$ is a P-basis of $\overline{\mathcal{B}^{\prime}}$ and, in particular, it is P-independent.

Before giving the main result of this section, we need another consequence of Theorem 4, which will allow us to define the concepts of complementary P-closed sets and complementary P-bases:

Corollary 37. Let $\Psi \subseteq \Omega \subseteq \mathbb{F}^{n}$ be P-closed sets. If $\Omega$ is finitely generated, then so is $\Psi$. Moreover, for any finite $P$-basis $\mathcal{B}$ of $\Psi$, there exists a finite $P$-independent set $\mathcal{C} \subseteq \Omega$ such that $\mathcal{B} \cap \mathcal{C}=\varnothing$ and $\mathcal{B} \cup \mathcal{C}$ is a P-basis of $\Omega$. In particular, if $\Phi=\overline{\mathcal{C}}$, then

$$
\operatorname{Rk}(\Omega)=\operatorname{Rk}(\Psi)+\operatorname{Rk}(\Phi) .
$$


Proof. Assume that $\Psi$ is not finitely generated. Using Lemma 36, we may construct iteratively a $\mathrm{P}$-independent set $\mathcal{D} \subseteq \Psi$ of size $\operatorname{Rk}(\Omega)+1$. This is absurd by the same argument as in the proof of Corollary 32 .

Now, we may extend $\mathcal{B}$ to a maximal P-independent subset of $\Omega$ by adding iteratively to it elements $\mathbf{c}_{1}, \mathbf{c}_{2}, \ldots, \mathbf{c}_{N} \in \Omega$, again by Lemma 36, which would be a P-basis of $\Omega$ by maximality (again by Lemma 36). By defining $\mathcal{C}=\left\{\mathbf{c}_{1}, \mathbf{c}_{2}, \ldots, \mathbf{c}_{N}\right\}$, the rest of the claims in the corollary follow.

Definition 38 (Complementary P-closed sets and P-bases). If $\Psi \subseteq \Omega \subseteq \mathbb{F}^{n}$ are finitely generated $\mathrm{P}$-closed sets and $\mathcal{B}$ and $\mathcal{C}$ are as in the previous Corollary, then we say that $\Phi=\overline{\mathcal{C}} \subseteq \Omega$ is a complementary P-closed set of $\Psi$ in $\Omega$, and $\mathcal{C}$ is a complementary P-basis of $\mathcal{B}$ in $\Omega$.

We may now state and prove the second main result of the paper, which describes the image and kernel of $E_{\Omega}^{\sigma, \delta}$ as left vector spaces over $\mathbb{F}$ with some particular left bases.

Theorem 5. Given a finitely generated P-closed set $\Omega \subseteq \mathbb{F}^{n}$ with finite $P$-basis $\mathcal{B}$, we have that

1. $\operatorname{Im}\left(E_{\Omega}^{\sigma, \delta}\right)$ is a left vector space over $\mathbb{F}$ of dimension $M=\operatorname{Rk}(\Omega)$ with left basis

$$
E_{\Omega}^{\sigma, \delta}\left(\mathcal{B}^{*}\right)=\left\{E_{\Omega}^{\sigma, \delta}\left(F_{1}\right), E_{\Omega}^{\sigma, \delta}\left(F_{2}\right), \ldots, E_{\Omega}^{\sigma, \delta}\left(F_{M}\right)\right\},
$$

where $\mathcal{B}^{*}=\left\{F_{1}, F_{2}, \ldots, F_{M}\right\}$ is a dual P-basis of $\mathcal{B}$. Observe that, by Corollary 31. $E_{\Omega}^{\sigma, \delta}\left(\mathcal{B}^{*}\right)$ depends only on $\mathcal{B}$ and not on the choice of the dual P-basis.

2. If $\mathbb{F}^{n}$ is finitely generated as $P$-closed set, $\mathcal{C}$ is a complementary $P$-basis of $\mathcal{B}$ in $\mathbb{F}^{n}$, and $\mathcal{C}^{*}=\left\{G_{1}, G_{2}, \ldots, G_{N}\right\}$ is a dual P-basis of $\mathcal{C}$ that is part of a dual P-basis $(\mathcal{B} \cup \mathcal{C})^{*}$ of $\mathcal{B} \cup \mathcal{C}$, then

$$
\operatorname{Ker}\left(E_{\Omega}^{\sigma, \delta}\right)=I\left(\mathbb{F}^{n}\right) \oplus\left\langle G_{1}, G_{2}, \ldots, G_{N}\right\rangle,
$$

as left vector spaces over $\mathbb{F}$, and $G_{1}, G_{2}, \ldots, G_{N}$ are left linearly independent over $\mathbb{F}$.

Proof. The proof of Item 1 was given in the proof of Corollary 32, Now we prove Item 2 :

First, $G_{1}, G_{2}, \ldots, G_{N}$ are left linearly independent over $\mathbb{F}$ by Item 1 , since so are their evaluations over $\Phi=\overline{\mathcal{C}}$.

Now we show that, if $F \in I\left(\mathbb{F}^{n}\right) \cap\left\langle G_{1}, G_{2}, \ldots, G_{N}\right\rangle$, then $F=0$. To that end, write $F=\sum_{i=1}^{N} \lambda_{i} G_{i}$, for some $\lambda_{i} \in \mathbb{F}$ and all $i=1,2, \ldots, N$. Since $F \in I\left(\mathbb{F}^{n}\right)$, it holds that $E_{\Phi}^{\sigma, \delta}(F)=0$, and since $E_{\Phi}^{\sigma, \delta}\left(G_{1}\right), E_{\Phi}^{\sigma, \delta}\left(G_{2}\right), \ldots, E_{\Phi}^{\sigma, \delta}\left(G_{M}\right)$ are left linearly independent by Item 1 , we conclude that $F=0$.

Next let $F \in \operatorname{Ker}\left(E_{\Omega}^{\sigma, \delta}\right)$. Then by Theorem 4, it holds that

$$
F-\sum_{i=1}^{N} F\left(\mathbf{c}_{i}\right) G_{i} \in I\left(\mathbb{F}^{n}\right),
$$


since this skew polynomial vanishes at $\mathcal{B} \cup \mathcal{C}$, and this set is a $\mathrm{P}$-basis of $\mathbb{F}^{n}$. Hence $F \in I\left(\mathbb{F}^{n}\right) \oplus\left\langle G_{1}, G_{2}, \ldots, G_{N}\right\rangle$.

Conversely, let $F \in I\left(\mathbb{F}^{n}\right) \oplus\left\langle G_{1}, G_{2}, \ldots, G_{N}\right\rangle$. By the assumptions, we have that $F(\mathbf{b})=0$, for all $\mathbf{b} \in \mathcal{B}$. Hence $F \in \operatorname{Ker}\left(E_{\Omega}^{\sigma, \delta}\right)$ again by Theorem 4 .

We conclude with the following consequence, which describes the quotient left modules over the ideal associated to a finitely generated P-closed set. Such quotient left modules include the minimal skew polynomial ring if $\mathbb{F}^{n}$ is finitely generated, which is the case if $\mathbb{F}$ is finite.

Corollary 39. If $\left\{F_{1}, F_{2}, \ldots, F_{M}\right\}$ is a dual P-basis of a finitely generated P-closed set $\Omega \subseteq \mathbb{F}^{n}$, then

$$
\mathbb{F}[\mathbf{x} ; \sigma, \delta] / I(\Omega) \cong\left\langle F_{1}, F_{2}, \ldots, F_{M}\right\rangle
$$

as left vector spaces, where the isomorphism is given by inverting the projection to the quotient ring. Moreover, $F_{1}, F_{2}, \ldots, F_{M}$ are left linearly independent, and hence

$$
\operatorname{dim}(\mathbb{F}[\mathbf{x} ; \sigma, \delta] / I(\Omega))=\operatorname{Rk}(\Omega) .
$$

In particular, the minimal skew polynomial ring $\mathbb{F}[\mathbf{x} ; \sigma, \delta] / I\left(\mathbb{F}^{n}\right)$ is a finite-dimensional left vector space over $\mathbb{F}$ of dimension $\operatorname{Rk}\left(\mathbb{F}^{n}\right)$ if $\mathbb{F}^{n}$ is finitely generated.

Proof. Again, it follows directly from Item 1 in Theorem 5 that $F_{1}, F_{2}, \ldots, F_{M}$ are left linearly independent over $\mathbb{F}$, since so are their evaluations over $\Omega$.

Now consider the left linear projection map $\rho:\left\langle F_{1}, F_{2}, \ldots, F_{M}\right\rangle \longrightarrow \mathbb{F}[\mathbf{x} ; \sigma, \delta] / I(\Omega)$. To show that it is onto, it suffices to observe that, given $F \in \mathbb{F}[\mathbf{x} ; \sigma, \delta]$, it holds that

$$
\rho(F)=\rho\left(\sum_{i=1}^{M} F\left(\mathbf{b}_{i}\right) F_{i}\right),
$$

by Item 1 in Theorem 4 , where $\mathcal{B}=\left\{\mathbf{b}_{1}, \mathbf{b}_{2}, \ldots, \mathbf{b}_{M}\right\}$ is the P-basis of $\Omega$ associated to $\left\{F_{1}, F_{2}, \ldots, F_{M}\right\}$.

Finally, the evaluation map $\mathbb{F}[\mathbf{x} ; \sigma, \delta] / I(\Omega) \longrightarrow \operatorname{Im}\left(E_{\Omega}^{\sigma, \delta}\right)$ is a left vector space isomorphism by definition, thus by Item 1 in Theorem 5 , it holds that

$$
\operatorname{dim}\left(\left\langle F_{1}, F_{2}, \ldots, F_{M}\right\rangle\right)=M=\operatorname{dim}\left(\operatorname{Im}\left(E_{\Omega}^{\sigma, \delta}\right)\right)=\operatorname{dim}(\mathbb{F}[\mathbf{x} ; \sigma, \delta] / I(\Omega)) .
$$

Hence $\rho$ is a left vector space isomorphism, and we are done.

As shown in Equation (14), if $\mathbb{F}$ is finite and has $q$ elements, then

$$
\operatorname{dim}\left(\mathbb{F}[\mathbf{x}] / I\left(\mathbb{F}^{n}\right)\right)=q^{n}=\operatorname{Rk}\left(\mathbb{F}^{n}\right),
$$

since $\operatorname{Rk}\left(\mathbb{F}^{n}\right)=\# \mathbb{F}^{n}=q^{n}$ in the conventional case. Hence the previous corollary extends this well-known result for finite fields. 


\section{Skew Vandermonde matrices and how to find P-bases}

In the univariate case $(n=1)$, Vandermonde matrices are a crucial tool to explicitly compute Lagrange interpolating polynomials. The multivariate case works similarly, although only existence of interpolating skew polynomials may be derived, and not their uniqueness. This is due to the non-square form of multivariate Vandermonde matrices.

In this section, we extend the concept of skew Vandermonde matrix from the univariate case in 10, 12 to the multivariate case. As applications and thanks to the recursive formula in Theorem 2, we show how to explicitly compute P-bases, dual P-bases and Lagrange interpolating skew polynomials over finitely generated P-closed sets.

The case $n=1$ in the following definition was given in [12, Eq. (4.1)], and previously in [10]:

Definition 40 (Skew Vandermonde matrices). Let $\mathcal{N} \subseteq \mathcal{M}$ be a finite set of monomials and let $\mathcal{B}=\left\{\mathbf{b}_{1}, \mathbf{b}_{2}, \ldots, \mathbf{b}_{M}\right\} \subseteq \mathbb{F}^{n}$. We define the corresponding $(\sigma, \delta)$-skew Vandermonde matrix, denoted by $V_{\mathcal{N}}^{\sigma, \delta}(\mathcal{B})$, as the $(\# \mathcal{N}) \times M$ matrix over $\mathbb{F}$ whose rows are given by

$$
\left(N_{\mathfrak{m}}\left(\mathbf{b}_{1}\right), N_{\mathfrak{m}}\left(\mathbf{b}_{2}\right), \ldots, N_{\mathfrak{m}}\left(\mathbf{b}_{M}\right)\right) \in \mathbb{F}^{M},
$$

for all $\mathfrak{m} \in \mathcal{N}$ (given certain ordering in $\mathcal{M}$ ). If $d$ is a positive integer, we define $\mathcal{M}_{d}$ as the set of monomials of degree less than $d$, and we denote

$$
V_{d}^{\sigma, \delta}(\mathcal{B})=V_{\mathcal{M}_{d}}^{\sigma, \delta}(\mathcal{B})
$$

An important consequence of Theorem 4 is finding the rank and a P-basis of a given finitely generated P-closed set:

Proposition 41. Given a finite set $\mathcal{G} \subseteq \mathbb{F}^{n}$ with $M$ elements, and $\Omega=\overline{\mathcal{G}}$, it holds that

$$
\operatorname{Rk}\left(V_{M}^{\sigma, \delta}(\mathcal{G})\right)=\operatorname{Rk}(\Omega)
$$

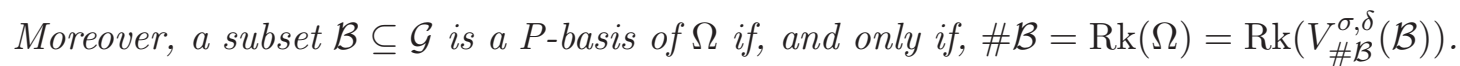

Hence applying Gaussian elimination to the matrix $V_{M}^{\sigma, \delta}(\mathcal{G})$, we may find the rank of $\Omega$ and at least one of its P-bases.

Proof. First, by Corollary 31 and Theorem [5, it holds that $\operatorname{Im}\left(E_{\Omega}^{\sigma, \delta}\right)$ is the left vector space generated by the evaluations $\left(N_{\mathfrak{m}}(\mathbf{a})\right)_{\mathbf{a} \in \Omega} \in \mathbb{F}^{\Omega}$, for $\mathfrak{m} \in \mathcal{M}_{M}$. By Lemma 35, to calculate $\operatorname{dim}\left(\operatorname{Im}\left(E_{\Omega}^{\sigma, \delta}\right)\right)$, we may restrict such evaluations to points in $\mathcal{G}$, and the first claim follows.

Now we prove the second claim. If $\mathcal{B}$ is a $\mathrm{P}$-basis of $\Omega$, then $\# \mathcal{B}=\operatorname{Rk}(\Omega)$ by definition, and $\operatorname{Rk}(\Omega)=\operatorname{Rk}\left(V_{\# \mathcal{B}}^{\sigma, \delta}(\mathcal{B})\right)$ by the first claim.

Conversely, if $\# \mathcal{B}=\operatorname{Rk}(\Omega)=\operatorname{Rk}\left(V_{\# \mathcal{B}}^{\sigma, \delta}(\mathcal{B})\right)$, then by Theorem 5 , it holds that

$$
\operatorname{dim}\left(\operatorname{Im}\left(E_{\Omega}^{\sigma, \delta}\right)\right)=\operatorname{Rk}(\Omega)=\operatorname{Rk}\left(V_{\# \mathcal{B}}^{\sigma, \delta}(\mathcal{B})\right) \leq \operatorname{dim}\left(\operatorname{Im}\left(E_{\mathcal{B}}^{\sigma, \delta}\right)\right) .
$$


Since the opposite inequality always holds, it follows from Lemma 35 that $\overline{\mathcal{B}}=\Omega$. Now, $\mathcal{B}$ is a minimal set of P-generators of $\Omega$, since $\# \mathcal{B}=\operatorname{Rk}(\Omega)$, all minimal sets of $\mathrm{P}$ generators are P-bases by Proposition 25 and all have the same size by Corollary 32 . Hence we conclude that $\mathcal{B}$ is a P-basis of $\Omega$.

A classical way of stating the Lagrange interpolation theorem is as the invertibility of Vandermonde matrices. This result is an immediate consequence of Theorem 4

Corollary 42. Let $\Omega \subseteq \mathbb{F}^{n}$ be a finitely generated P-closed set with P-basis $\mathcal{B}=$ $\left\{\mathbf{b}_{1}, \mathbf{b}_{2}, \ldots, \mathbf{b}_{M}\right\}$. There exists a solution to the linear system

$$
\left(F_{\mathfrak{m}}\right)_{\mathfrak{m} \in \mathcal{M}_{M}} V_{M}^{\sigma, \delta}(\mathcal{B})=\left(a_{1}, a_{2}, \ldots, a_{M}\right),
$$

for any $a_{1}, a_{2}, \ldots, a_{M} \in \mathbb{F}$ (that is, $V_{M}^{\sigma, \delta}(\mathcal{B})$ is left invertible). For any solution, the corresponding skew polynomial $F=\sum_{\mathfrak{m} \in \mathcal{M}_{M}} F_{\mathfrak{m}} \mathfrak{m}$ satisfies that $F\left(\mathbf{b}_{i}\right)=a_{i}$, for $i=$ $1,2, \ldots, M$, and $\operatorname{deg}(F)<M$.

Another important immediate consequence is the following:

Corollary 43. Given a P-basis $\mathcal{B}$, with $M$ elements, of a P-closed set, one can obtain a dual P-basis of $\mathcal{B}$, consisting of skew polynomials of degree less than $M$, by solving $M$ systems of $M$ linear equations whose coefficients are taken from left linearly independent rows in $V_{M}^{\sigma, \delta}(\mathcal{B})$.

In conclusion, to find a P-basis of a P-closed set $\Omega \subseteq \mathbb{F}^{n}$ with $M=\operatorname{Rk}(\Omega)$ and generated by a finite set $\mathcal{G}$, we need to find $M$ linearly independent columns in $V_{M}^{\sigma, \delta}(\mathcal{G})$. Using Gaussian elimination, such method has exponential complexity in $M$ if $n>1$, since the number of rows in $V_{M}^{\sigma, \delta}(\mathcal{G})$ is $\# \mathcal{M}_{M}$, which is exponential in $M$.

Fortunately, if we are given or have precomputed a P-basis of $\Omega$, we may find Lagrange interpolating skew polynomials over $\Omega$ with complexity $\mathcal{O}\left(M^{3}\right)$, and find a dual P-basis with complexity $\mathcal{O}\left(M^{4}\right)$.

\section{Conclusion and open problems}

In this paper, we have introduced free multivariate skew polynomials with coefficients over rings, although we have focused on division rings. We have given a natural definition of evaluation (Definition 9), which extends the univariate case studied in [10, 11, 12, and we have obtained a product rule (Theorem 3). With these notions and assumptions, we were able to define general nonfree multivariate skew polynomial rings (Definition 19), where evaluation is still natural. We have described (by giving dimensions and left bases) in Theorem 5 the left vector spaces of functions defined by multivariate skew polynomials, when defined over a finitely generated P-closed set (set of zeros). This has been done thanks to a Lagrange-type interpolation theorem (Theorem 4). The following problems are left open: 
1. Find explicit descriptions of general multivariate skew polynomial rings. In other words, find explicit descriptions of matrix morphisms, vector derivations and twosided ideals contained in $I\left(\mathbb{F}^{n}\right)$.

2. The previous item is particularly interesting in the case of finite fields, where the minimal skew polynomial ring is generated by a finite collection of skew polynomials.

3. Although we have given computational methods to find ranks, P-bases, dual Pbases and Lagrange interpolating skew polynomials, it would be interesting to obtain explicit formulas for such objects. Algorithms for finding P-bases with polynomial complexity are also interesting, as well as reducing the complexity of finding Lagrange interpolating skew polynomials.

4. Investigate how to perform Euclidean-type divisions over multivariate skew polynomial rings, which would extend Lemma 5. A notion of Gröbner basis may be possible and useful in this context.

\section{Acknowledgement}

The authors wish to thank the anonymous reviewer for their very helpful comments. The first author gratefully acknowledges the support from The Independent Research Fund Denmark (Grant No. DFF-4002-00367, Grant No. DFF-5137-00076B "EliteForskRejsestipendium", and Grant No. DFF-7027-00053B).

\section{References}

[1] S. A. Amitsur and L. W. Small. Polynomials over division rings. Israel Journal of Mathematics, 31(3):353-358, Sep 1978.

[2] D. Boucher, W. Geiselmann, and F. Ulmer. Skew-cyclic codes. Applicable Algebra in Engineering, Communication and Computing, 18(4):379-389, 2007.

[3] P. M. Cohn. Free rings and their relations. London: Academic Press, 1971.

[4] A. Lj. Erić. Polynomial interpolation problem for skew polynomials. Applicable Analysis and Discrete Mathematics, 1(2):403-414, 2007.

[5] E. M. Gabidulin. Theory of codes with maximum rank distance. Problems Information Transmission, 21(1):1-12, 1985.

[6] A. Galligo. Some algorithmic questions on ideals of differential operators. In Proc. EUROCAL '85, pages 413-421. Springer Berlin Heidelberg, 1985.

[7] W. Geiselmann and F. Ulmer. Skew Reed-Muller codes. Preprint available at: https://hal.archives-ouvertes.fr/hal-01633128v3, November 2017.

[8] A. V. Jategaonkar. A counter-example in ring theory and homological algebra. Journal of Algebra, 12(3):418-440, 1969. 
[9] A. Kandri-Rody and V. Weispfenning. Non-commutative Gröbner bases in algebras of solvable type. Journal of Symbolic Computation, 9(1):1-26, January 1990.

[10] T. Y. Lam. A general theory of Vandermonde matrices. Expositiones Mathematicae, 4:193$215,1986$.

[11] T. Y. Lam and A. Leroy. Algebraic conjugacy classes and skew polynomial rings. In Perspectives in Ring Theory, pages 153-203. Springer, 1988.

[12] T. Y. Lam and A. Leroy. Vandermonde and Wronskian matrices over division rings. Journal of Algebra, 119(2):308-336, 1988.

[13] T. Y. Lam and A. Leroy. Hilbert 90 theorems over divison rings. Transactions of the American Mathematical Society, 345(2):595-622, 1994.

[14] H. W. Lenstra. Lectures on Euclidean rings. Universität Bielefeld, 1974.

[15] A. Leroy. Pseudolinear transformations and evaluation in Ore extensions. Bulletin of the Belgian Mathematical Society, 2(3):321-347, 1995.

[16] S. Liu, F. Manganiello, and F. R. Kschischang. Kötter interpolation in skew polynomial rings. Designs, Codes and Cryptography, 72(3):593-608, September 2014.

[17] J. H. Maclagan-Wedderburn. A theorem on finite algebras. Transactions of the American Mathematical Society, 6(3):349-352, 1905.

[18] U. Martínez-Peñas. Linearized multivariate skew polynomials and Hilbert 90 theorems with multivariate norms. In Proc. XVI EACA, Zaragoza - Encuentros de Álgebra Computacional y Aplicaciones, pages 119-122, 2018.

[19] U. Martínez-Peñas. Skew and linearized Reed-Solomon codes and maximum sum rank distance codes over any division ring. Journal of Algebra, 504:587-612, 2018.

[20] O. Ore. Theory of non-commutative polynomials. Annals of Mathematics (2), 34(3):480$508,1933$.

[21] J. G. Oxley. Matroid Theory. Oxford graduate texts in mathematics. Oxford University Press, 2006.

[22] Y. Zhang. A secret sharing scheme via skew polynomials. In Proc. 2010 International Conference on Computational Science and Its Applications, pages 33-38, March 2010.

[23] X. Zhao and Y. Zhang. A signature-based algorithm for computing Gröbner-Shirshov bases in skew solvable polynomial rings. Open Mathematics, 13(1):298-307, 2015. 